\title{
Magnetic configurations of the tilted current sheets in magnetotail
}

\author{
C. Shen ${ }^{1}$, Z. J. Rong ${ }^{1,2}$, X. Li ${ }^{3}$, M. Dunlop ${ }^{4,5}$, Z. X. Liu ${ }^{1}$, H. V. Malova ${ }^{6}$, E. Lucek ${ }^{5}$, and C. Carr ${ }^{5}$ \\ ${ }^{1}$ State Key Laboratory of Space Weather, Center for Space Science and Applied Research, Chinese Academy of Sciences, \\ Beijing 100190, China \\ ${ }^{2}$ College of Earth Science, Graduate University of Chinese Academy of Sciences, Beijing, 100049, China \\ ${ }^{3}$ Laboratory for Atmosphere and Space Physics, University of Colorado, Boulder, USA \\ ${ }^{4}$ Rutherford Appleton Laboratory, Chilton, DIDCOT, Oxfordshire OX11 0QX, UK \\ ${ }^{5}$ Imperial College of Science, Technology and Medicine, London, UK \\ ${ }^{6}$ Nuclear Physics Institute, Moscow State University, 119899 Moscow, Russia
}

Received: 7 May 2008 - Revised: 15 August 2008 - Accepted: 29 September 2008 - Published: 17 November 2008

\begin{abstract}
In this research, the geometrical structures of tilted current sheet and tail flapping waves have been analysed based on multiple spacecraft measurements and some features of the tilted current sheets have been made clear for the first time. The geometrical features of the tilted current sheet revealed in this investigation are as follows: (1) The magnetic field lines (MFLs) in the tilted current sheet are generally plane curves and the osculating planes in which the MFLs lie are about vertical to the equatorial plane, while the normal of the tilted current sheet leans severely to the dawn or dusk side. (2) The tilted current sheet may become very thin, the half thickness of its neutral sheet is generally much less than the minimum radius of the curvature of the MFLs. (3) In the neutral sheet, the field-aligned current density becomes very large and has a maximum value at the center of the current sheet. (4) In some cases, the current density is a bifurcated one, and the two humps of the current density often superpose two peaks in the gradient of magnetic strength, indicating that the magnetic gradient drift current is possibly responsible for the formation of the two humps of the current density in some tilted current sheets. Tilted current sheets often appear along with tail current sheet flapping waves. It is found that, in the tail flapping current sheets, the minimum curvature radius of the MFLs in the current sheet is rather large with values around $1 R_{E}$, while the neutral sheet may be very thin, with its half thickness being several tenths of $R_{E}$. During the flapping waves, the current sheet is tilted substantially, and the maximum tilt angle is generally larger than $45^{\circ}$. The phase velocities of these flapping waves are several tens $\mathrm{km} / \mathrm{s}$, while their periods and wavelengths are several tens of minutes, and several earth radii, respectively.
\end{abstract}

Correspondence to: C. Shen

(sc@ cssar.ac.cn)
These tail flapping events generally last several hours and occur during quiet periods or periods of weak magnetospheric activity.

Keywords. Magnetospheric physics (Magnetospheric configuration and dynamics; Magnetotai; Magnetotail boundary layers) - Space plasma physics (Discontinuities)

\section{Introduction}

Since the discovery of the magnetotail neutral sheet (NS) (Ness, 1965), which is the region where the magnetic field reverses its direction, extensive investigations have been made in this field. One significant feature of the tail current sheet (CS) is that it may have flapping motion in the north-south direction (Speiser and Ness, 1967; Toichi and Miyazaki, 1976; Lui et al., 1978). This flapping motion of the current sheet may have a wavy profile either along the tail direction (Speiser, 1973) or along the dawn-dusk direction (Lui et al., 1978, 1984). Some attempts have been made to study the origin and natures of the CS flapping with single spacecraft or two spacecraft measurements (e.g. Toyichi and Miyasaki, 1976; Fairfield et al., 1981; McComas et al., 1986; Sergeev et al., 1993). Toichi and Miyazaki (1976) have argued that the tail flapping motion is possibly caused by the penetration of IMF Alfvénic waves into magnetotail or the Pc5 geomagnetic pulsation. The four spacecraft measurements of Cluster mission (Escoubet et al., 2001) can separate the spatial and temporary variations of physical processes so as to provide more opportunities to explore the natures of the complicated tail current sheet. Zhang et al. (2002), Runov et al. (2003), and Sergeev et al. (2003, 2004), have reported on the low-frequency oscillations of the tail current sheet which

Published by Copernicus Publications on behalf of the European Geosciences Union. 
they have argued are generated by some impulsive source in the center of tail and propagating toward the flanks with velocities of a few tens $\mathrm{km} / \mathrm{s}$. Joint observations of Double Star Project (Liu et al., 2005) and Cluster (Escoubet et al., 2001) revealed that kink-like waves along the Y-axis can be observed at the geocentric distance of $11-19 R_{E}$ (Zhang et al., 2005). The locations of the occurrence of the tail flapping motion may be in the range $-11 R_{E}>\mathrm{X}>-30 R_{E}$ (Lui et al., 1978; Sergeev et al., 1993, 2006; Runov et al., 2005a). Sergeev et al. (2006) have found statistically that, similar to BBFs, current sheet flapping events tend to occur at the center of tail, but these two processes have no one-to-one local correspondence. Some theoretical explanations to the causes of CS flapping motion have been proposed (Nakagawa and Nishida, 1989; Golovchanskaya and Maltsev, 2005; Malova et al., 2007; Erkaev et al., 2008). Nakagawa and Nishida (1989) have suggested that the kink-like waves propagating towards the flanks might be excited by the Kelvin-Helmholtz instability at the plasma sheet. Golovchanskaya and Maltsev (2005) have argued the flapping motions could be the eigen oscillation of the plasma confined in the curvilinear magnetic field in the plasma sheet. Malova et al. (2007) have speculated the flapping wave might be induced by the asymmetric ion flow in the Northern and Southern Hemispheres. Erkaev et al. (2008) have interpreted it as a new kind of MHD wave related to a coexistence of two gradients of the tangential and normal magnetic field components of the current sheet.

The features of the tilted CS associated with the tail CS flapping motion have drawn much attention and are still under investigation. For the conventional tail current sheet (Lui, 2004, and the references therein), its normal is approximately along the south-north direction and the current sheet is almost parallel to the equatorial plane. The osculating planes of the magnetic field lines (MFLs) in the CS are about vertical to the equatorial plane (Shen et al., 2003) and the current inside of the CS is duskward and also cross-wise to the field. We may call this type of CS a normal current sheet. Sometimes, the normal current sheet may be approximated with Harris model (Harris, 1962; Lui, 2004; Thompson et al., 2005). The tail CS flapping motion may change the orientation of the current sheet and lead to the formation of the tilted current sheet (Sergeev et al., 2003, 2004; Volwerk et al., 2003; Runov et al., 2005a, b, 2006; Nakamura et al., 2006; Petrukovich et al., 2006). For the tilted current sheet associated with the tail flapping motion, its normal deviates from the northern direction and sometimes the tilted current sheet can be nearly vertical to the equatorial plane. Petrukovich et al. (2006) have argued that the tilted current sheet is caused by the slippage of the magnetic flux tubes in the flapping plasma sheet and found that the magnetic amplitude and magnetic gradient are approximately proportional to the steepness of the current sheet.

Nevertheless the 3-D magnetic geometry of the flapping current sheet is still not so clear. Obviously, in order to resolve this issue, new analysis methods should be employed to better reveal the detailed quantitative properties of the tilted current sheet. Here we will take advantage of Cluster multiple-point observations to quantitatively and explicitly investigate the features of the tilted current sheets, such as the geometric structure of the MFLs (including their curvature, binormal, etc.), the orientation of the current sheet, and the current density distribution in the tilted current sheets. The nature of tail flapping motions has also been explored and their possible mechanism has been given.

\section{Analysis on the magnetic features of tilted current sheet}

The four-point observations of Cluster have offered an excellent and unprecedented opportunity to study magnetospheric dynamics (Escoubet et al., 2001). With Cluster magnetic measurements (Balogh et al., 2001) and the previously developed multiple point data analysis methods (Chanteur, 1998; Harvey, 1998; Dunlop and Woodward, 1998; Dunlop et al., 2002; Shen et al., 2003, 2007a, b, 2008a, b; Shi et al., 2005, 2006; Sonnerup et al., 2008), the current density, magnetic gradient and magnetic field geometry, and motion of the sheet, can be found. The actual structures of the tail current sheet can often be revealed as distinct from the temporal variations.

To illustrate the main features of magnetic geometry of the tilted current sheet, two typical cases have been studied in details in Sect. 2.1. In Sect. 2.2, the properties of the tail flapping waves have been investigated.

\subsection{Features of the tilted current sheet}

In this section, we will analyse two single tilted current sheet crossing events on 5 August 2001 and 3 August 2004 and uncover the general geometrical features of this kind of current sheet by using newly developed, multi-point data analysis approaches (Shen et al., 2003, 2007a, b, 2008a). In this study, the magnetic field data of the Cluster four spacecraft have a time resolution of $4 \mathrm{~s}$, where GSM coordinates are used here. It is useful to define the spherical coordinate for any vector direction $\theta, \varphi)$ in the frame of GSM. The coordinate $\theta\left(0^{\circ} \leq \theta \leq 180^{\circ}\right)$ is the angle between the positive $\mathrm{z}$-axis and the vector direction, and referred to as the polar angle; The coordinate $\phi\left(0^{\circ} \leq \phi<360^{\circ}\right)$ is the angle between the positive $\mathrm{x}$-axis and the line from the vector direction projected onto the xy-plane, and referred to as the azimuth angle. For example, the dawn direction or $-\mathrm{y}$-direction is at $\theta=90^{\circ}$ and $\varphi=270^{\circ}$, while the dusk direction or $+\mathrm{y}$ direction is at $\theta=90^{\circ}$ and $\varphi=90^{\circ}$.

\subsubsection{Case on 5 August 2001}

We first investigate the crossing event of current sheet on 5 August 2001 around 17:42 17:47 UT. During this event, the upstream interplanetary magnetic field (IMF) is northward, 


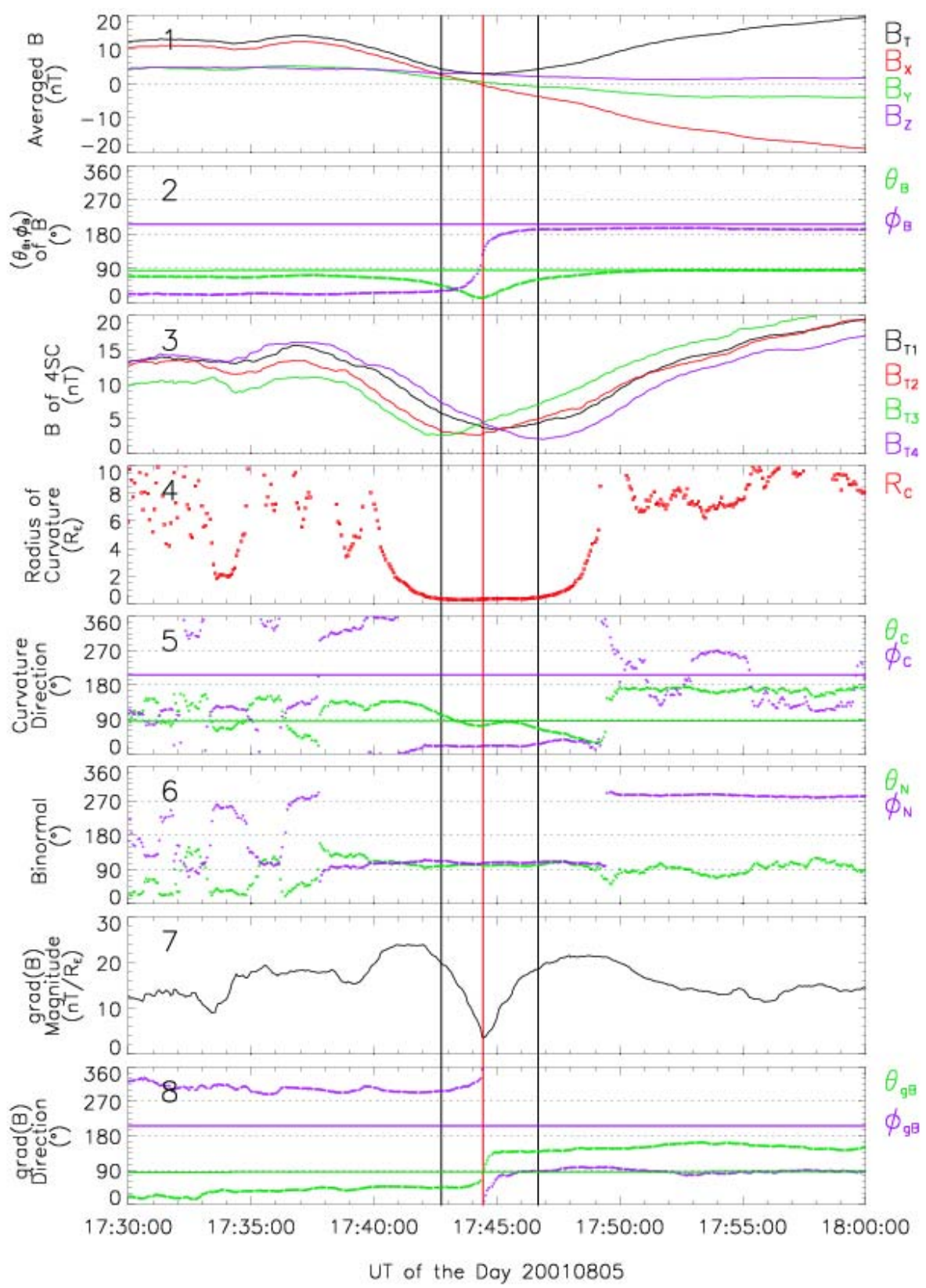

Fig. 1a. The geometric structure of the tilted current sheet during one Cluster crossing event on 5 August 2001. Panel 1: the magnetic field at the center of Cluster tetrahedron as are the average of those at the four spacecraft; panel 2: the directional angle $\left(\theta_{B}, \varphi_{B}\right)$ of the magnetic field at the center of Cluster tetrahedron; panel 3: magnetic strengths observed by the 4 spacecraft of Cluster; panel 4: the radius of curvature $R_{c}$; panel 5: the direction angles $\left(\theta_{c}, \varphi_{c}\right)$ of the curvature of the MFLs, that is also the direction angles of the principal normal; panel 6: the direction angles $\left(\theta_{N}, \varphi_{N}\right)$ of the normal of the osculating plane, or the binormal, of the MFLs; panel 7: the value of the gradient of magnetic field strength; panel 8: the directional angle $\left(\theta_{g B}, \varphi_{g B}\right)$ of the gradient of magnetic field strength. GSM coordinates are used here. The polar angle and azimuthal angle of the position vector of Cluster in GSM are also plotted in the panel of $\nabla B / B$ direction as green and violet horizontal lines, respectively.

and the magnetospheric activity is very weak with a rather low AE index. At the time of this event, Cluster is about 19.3 $R_{E}$ away from the Earth center and the polar angle and azimuthal angles of its position in GSM are $(\theta, \varphi)=\left(85^{\circ}\right.$, $206^{\circ}$ ), respectively. The Cluster tetrahedron was nearly reg- ular, and the averaged distance between its nodes and the barycenter is about $1200 \mathrm{~km}$.

The magnetic structure of this current sheet is shown in Fig. 1a and b. Panels 1-3 of Fig. 1a show the variation of magnetic field as the spacecraft cross the tilted current sheet. 


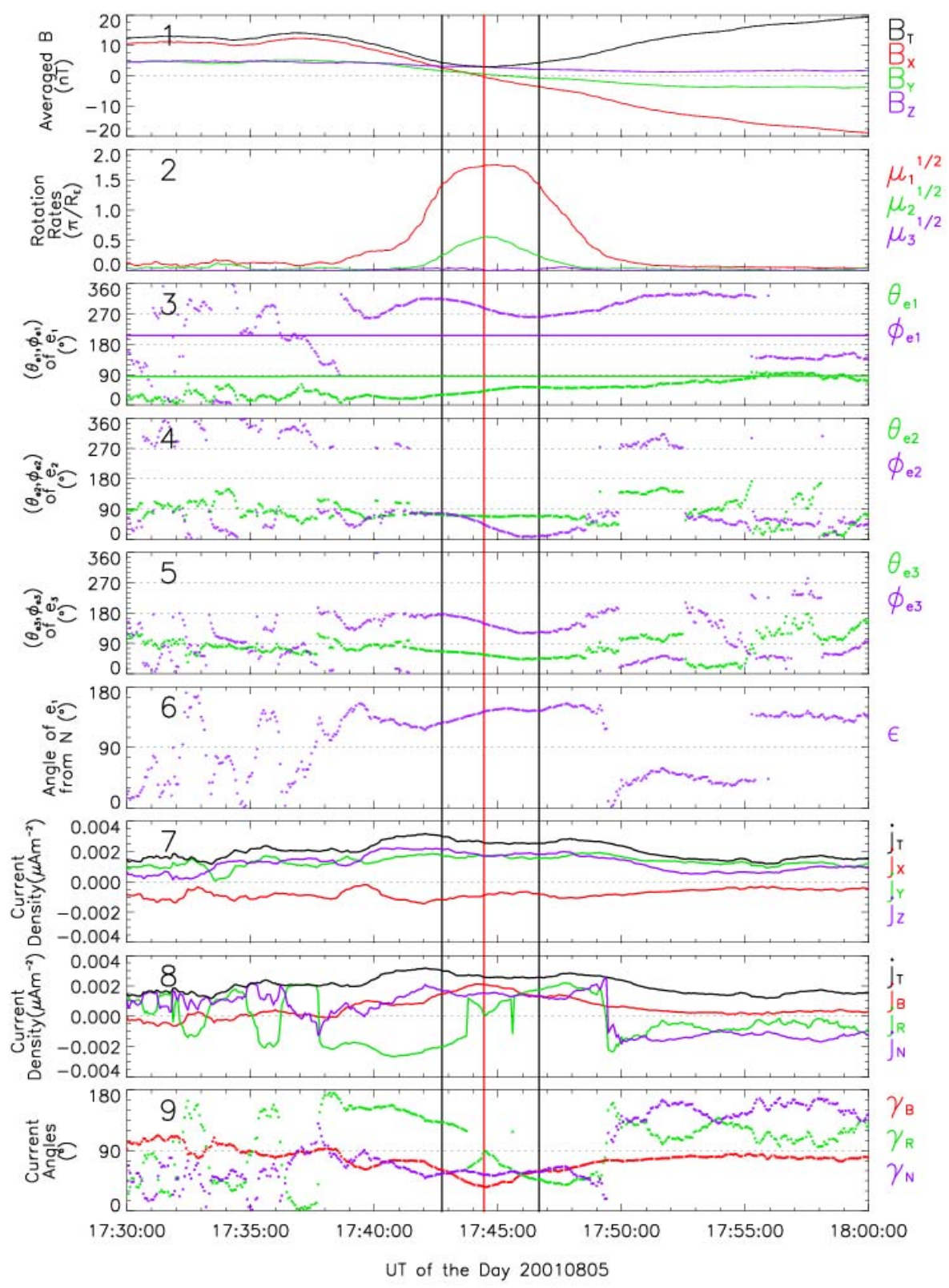

Fig. 1b. The geometric structure of the tilted current sheet during one Cluster crossing event on 5 August 2001. Panel 1: the magnetic field at the center of Cluster tetrahedron as are the average of those at the four spacecraft; panel 2: the maximum, medium and minimum rotation rate of the magnetic field; panel 3: the directional angles $\left(\theta_{e 1}, \varphi_{e 1}\right)$ of the first eigenvector $\hat{\boldsymbol{e}}^{(1)}$; panel 4: the directional angles $\left(\theta_{e 2}, \varphi_{e 2}\right)$ of the second eigenvector $\hat{\boldsymbol{e}}^{(2)}$; panel 5: the directional angles $\left(\theta_{e 3}, \varphi_{e 3}\right)$ of the third eigenvector $\hat{\boldsymbol{e}}^{(3)}$; panel 6: the angle $\varepsilon$ between the first eigenvector $\hat{\boldsymbol{e}}^{(1)}$ and the binormal of the MFLs; panel 7: The three components $\left(j_{x}, j_{y}, j_{z}\right)$ of the current density at GSM coordinates; panel 8: The three components $\left(j_{B}, j_{R}, j_{N}\right)$ of the current density at the local natural coordinates, where $j_{B}$ is the field-aligned component, $j_{R}$ the component along the curvature and $j_{N}$ along the binormal; panel 9: the angle $\left(\gamma_{B}, \gamma_{R}, \gamma_{N}\right)$ between current density and magnetic field, curvature and binormal of the MFLs, respectively. GSM coordinates are used here. The polar angle and azimuthal angle of the position vector of Cluster in GSM are also plotted in the two panels of $\nabla B / B$ direction and $\hat{\boldsymbol{e}}^{(1)}$ direction as green and violet horizontal lines, respectively.

When Cluster crosses the tail current sheet from the Northern Hemisphere into the Southern Hemisphere, the measured magnetic field vector points earthward at northern side, then northward at the center of the current sheet (its polar angle
$\theta_{B} \approx 15^{\circ}$ ), and finally tailward at the southern side, manifesting the typical features of the MFLs of current sheet. At the center of the current sheet, the magnetic strength drops to the minimum value of $B_{\mathrm{min}} \approx 2.9 \mathrm{nT}$. According to earlier 
investigations (Shen et al., 2007a), the neutral sheet (NS) can be defined as the region within which $B \leq \sqrt{2} B_{\min }$, where $B_{\min }$ is the minimum value of the magnetic strength at the center of the current sheet. Within the NS defined above, the magnetic field vector rotates about $90^{\circ}$ totally from one boundary to another of the NS (Shen et al., 2008b). Because the main rotation of the magnetic vector occurs within the NS, we may regard the NS as the magnetic vector rotation layer in the current sheet. In Fig. 1a, the boundaries of the neutral sheet are marked by two vertical black lines, and the center of the neutral sheet/current sheet is denoted by a vertical red line.

Panels 4-6 of Fig. 1a show the features of the geometry of the MFLs in the current sheet. As seen in panel 4, the minimum curvature radius of the MFLs appears in NS, that is $\sim 0.28 R_{E}=1784 \mathrm{~km}$. Note that the magnetic unit vector $\hat{\boldsymbol{b}}(\hat{\boldsymbol{b}}=\boldsymbol{B} / B)$, curvature $\boldsymbol{\rho}_{c}\left(\boldsymbol{\rho}_{c}=(\hat{\boldsymbol{b}} \cdot \nabla) \hat{\boldsymbol{b}}\right)$, and the binormal $\hat{\boldsymbol{N}}\left(\hat{\boldsymbol{N}}=\hat{\boldsymbol{b}} \times \boldsymbol{\rho}_{c} /\left|\hat{\boldsymbol{b}} \times \boldsymbol{\rho}_{c}\right|\right)$ are orthogonal to each other (Shen et al., 2003). Panels 5 and 6 show that the curvature points towards earth when approaching NS, while the direction of binormal in the NS is duskward continuously with its polar angle and azimuthal angles $\left(\theta_{N}, \varphi_{N}\right)=\left(102^{\circ}, 107^{\circ}\right)$, implying that the MFLs of the current sheet are almost plane curves and their osculating planes vertical to the equatorial plane. The topology of a single magnetic field line is nearly the same as that in a normal current sheet (see the Fig. 5 of Shen et al., 2003).

Panels 7-8 of Fig. 1a show the properties of the gradient of the magnetic strength. The direction of the magnetic gradient can reflect the normal of the CS approximately (Shen et al., 2003, 2007b, 2008). Panels 7 and 8 show that $\hat{\boldsymbol{n}}_{g B}$ (i.e. the direction of $\nabla B)$ is $\left(46^{\circ}, 298^{\circ}\right)$ at the northern side $\left(B_{x}>0\right)$ and $\left(139^{\circ}, 87^{\circ}\right)$ at the southern side $\left(B_{x}<0\right)$, yielding that the normal $\hat{\boldsymbol{n}}$ of the CS/NS is approximately $\left(44^{\circ}, 283^{\circ}\right)$. In addition, at the center of the current sheet $\nabla B$ reverses its direction and its value minimizes.

We may estimate the thickness of the current sheet (Shen, 2007) based on the knowledge of $\nabla B$ crossing the current sheet yielded from Cluster multi-point measurements. The half thickness, $H$, of the current sheet may be expressed as

$H=\int_{0}^{H} d Z=\int_{B_{\min }}^{B_{0}} d B /|\nabla B| \approx\left(B_{0}-B_{\min }\right) /\langle|\nabla B|\rangle$,

where $B_{0}$ is the magnetic strength in the lobes, and $\langle|\nabla B|\rangle$ is the average of $|\nabla B|$. For this current sheet, $B_{0} \approx 18 \mathrm{nT}$, $B_{\text {min }} \approx 2.9 \mathrm{nT}$, and $\langle|\nabla B|\rangle \approx 18 \mathrm{nT} / R_{E}$. Thus from Eq. (1) we get the half thickness of this current sheet as $H \approx 0.8 R_{E}$.

Panels 2-6 of Fig. 1b show the other features of the magnetic rotation. Panel 2 shows that the main rotations of the magnetic field occur within the neutral sheet. In order to measure the spatial rotation of the magnetic vector quantitatively, we have developed the magnetic rotation analysis (MRA) method (Shen et al., 2007a, 2008). Based on 4 point magnetic field measurements, MRA yields that the magnetic vector $\boldsymbol{B}$ has the maximum rotation rate $\mu_{1}^{1 / 2}$ along $\hat{\boldsymbol{e}}^{(1)}$, the median rotation rate $\mu_{2}^{1 / 2}$ along $\hat{\boldsymbol{e}}^{(2)}$ and the minimum rotation rate $\mu_{3}^{1 / 2}$ along $\hat{\boldsymbol{e}}^{(3)}$, where $\hat{\boldsymbol{e}}^{(1)}, \hat{\boldsymbol{e}}^{(2)}$ and $\hat{\boldsymbol{e}}^{(3)}$ are three characteristic eigenvectors of the magnetic field. For an ideal, one-dimensional boundary, e.g. one current sheet, its normal is just parallel to the first eigenvector $\hat{\boldsymbol{e}}^{(1)}$, and along the normal direction the magnetic vector has the maximum rotation rate $\mu_{1}^{1 / 2}$ (Shen et al., 2007a, 2008). It can be seen in panel 2 of Fig. $1 \mathrm{~b}$ that, the maximum rotation rate of the magnetic field vector is $\mu_{1}^{1 / 2} \approx 1.8 \pi / R_{E}$, much larger than the medium rotation rate $\mu_{2}^{1 / 2}$ and the minimum rotation rate $\mu_{3}^{1 / 2}$, implying that this tilted CS is approximately a 1-D structure with $B_{y}$ and $B_{z}$ being constant approximately. By using the previously developed method (Shen et al., 2007a), the half thickness of the one-dimensional NS can be estimated from the magnetic rotation rate as

$h \approx 1 / \mu_{1 \max }^{1 / 2}$.

For this current sheet, the largest maximum rotation rate $\mu_{1 \max }^{1 / 2} \approx 1.8 \pi / R_{E}$; therefore, the half thickness of this NS is $h \approx 1 / \mu_{1 \max }^{1 / 2} \approx\left(1.8 \pi / R_{E}\right)^{-1} \approx 1130 \mathrm{~km} \approx 0.18 R_{E}$. Another approach for determining the half thickness of the onedimensional NS is based on the Ampere's Law (Shen et al., 2008 b), that is

$h_{j}=\frac{B_{\min }}{\mu_{0}\langle j\rangle}$,

where $\langle j\rangle$ is the averaged current density within the NS. In this NS, $B_{\min } \approx 2.9 \mathrm{nT},\langle j\rangle \approx 0.0026 \mu \mathrm{A} / \mathrm{m}^{2}$, thus the half thickness of the NS is $h_{j} \approx B_{\min } /\left(\mu_{0}\langle j\rangle\right) \approx 890 \mathrm{~km} \approx 0.14 R_{E}$. Thus, the half thicknesses of the NS yielded from the two different methods are very near. Obviously, the half thickness of the NS is much less than the half-thickness $H\left(0.8 R_{E}\right.$ as above deduced) of the CS. The eigenvector direction of $\mu_{1}, \mu_{2}, \mu_{3}$ are shown in panels 3-5, respectively. The first eigenvector $\boldsymbol{e}^{(1)}$ is $\left(\theta_{e 1}, \varphi_{e 1}\right) \approx\left(42^{\circ}, 287^{\circ}\right)$ at the center of the NS, which is the normal direction of this tilted CS (Shen et al., 2007a). This is consistent with the judgment from the magnetic gradient as above. Thus, this tilted CS leans to the dawn side by about $42^{\circ}$.

The velocity of the NS along the normal direction relative to the spacecraft could be estimated as $V_{n} \approx \frac{2 h}{\Delta t}=\frac{2 \times 1127 \mathrm{~km}}{221 \mathrm{~s}} \approx 10.2 \mathrm{~km} / \mathrm{s}$, where $\Delta t$ is the time for Cluster crossing the neutral sheet. This result also agrees with that estimated by curvature investigation method $(\sim 9 \mathrm{~km} / \mathrm{s})$ (Shen et al., 2003). Due to the much smaller velocity of the spacecraft $(\sim 1.1 \mathrm{~km} / \mathrm{s})$, it is reasonable to interpret $V_{n}$ as the absolute normal flapping velocity.

It will be meaningful to define clearly one parameter to measure the slipping between the magnetic field lines in the tilted current sheet. Here we may define the slipping angle (or tilt angle) $\delta$ of the tilted current sheet as the angle between 
Table 1. The parameters in the center of the NS of the two TCS.

\begin{tabular}{|c|c|c|c|c|c|c|c|c|c|c|c|c|c|c|c|}
\hline $\begin{array}{l}\text { TCS } \\
\text { event }\end{array}$ & $\begin{array}{c}R \\
\left(R_{E}\right)\end{array}$ & $\begin{array}{c}(\theta, \varphi) \\
\text { in GSM }\end{array}$ & $\begin{array}{l}B_{\min } \\
(\mathrm{nT})\end{array}$ & $\hat{b}$ & $\hat{N}$ & $\hat{\boldsymbol{e}}^{(1)}(\hat{\boldsymbol{n}})$ & $\begin{array}{c}\hat{\boldsymbol{n}} \text { by } \\
\nabla B /|\nabla B|\end{array}$ & $\begin{array}{c}R_{c, \min } \\
\left(R_{E}\right)\end{array}$ & $\begin{array}{c}\mu_{1 \max }^{1 / 2} \\
\left(\pi / R_{E}\right)\end{array}$ & $\begin{array}{c}j \\
\left(\mu A / m^{2}\right)\end{array}$ & $\gamma_{B}$ & $\gamma_{N}$ & $\begin{array}{c}\delta \approx \\
\left|90^{\circ}-\varepsilon\right|\end{array}$ & $\begin{array}{c}h \\
\left(R_{E}\right)\end{array}$ & $\begin{array}{c}h_{j} \\
\left(R_{E}\right)\end{array}$ \\
\hline $\begin{array}{l}5 \text { Aug } \\
2001\end{array}$ & 19.3 & $\left(85^{\circ}, 206^{\circ}\right)$ & 2.9 & $\left(13^{\circ}, 103^{\circ}\right)$ & $\left(102^{\circ}, 107^{\circ}\right)$ & $\left(42^{\circ}, 287^{\circ}\right)$ & $\left(44^{\circ}, 283^{\circ}\right)$ & 0.31 & 1.7 & 0.0026 & $36^{\circ}$ & $54^{\circ}$ & $51^{\circ}$ & 0.18 & 0.14 \\
\hline $\begin{array}{l}3 \text { Aug } \\
2004\end{array}$ & 19.1 & $\left(87^{\circ}, 211^{\circ}\right)$ & 7.7 & $\left(8.7^{\circ}, 269^{\circ}\right)$ & $\left(83^{\circ}, 114^{\circ}\right)$ & $\left(89^{\circ}, 115^{\circ}\right)$ & $\left(89.6^{\circ}, 111^{\circ}\right)$ & 1.8 & 1.6 & 0.0047 & $16^{\circ}$ & $75^{\circ}$ & $83.3^{\circ}$ & 0.20 & 0.20 \\
\hline
\end{tabular}
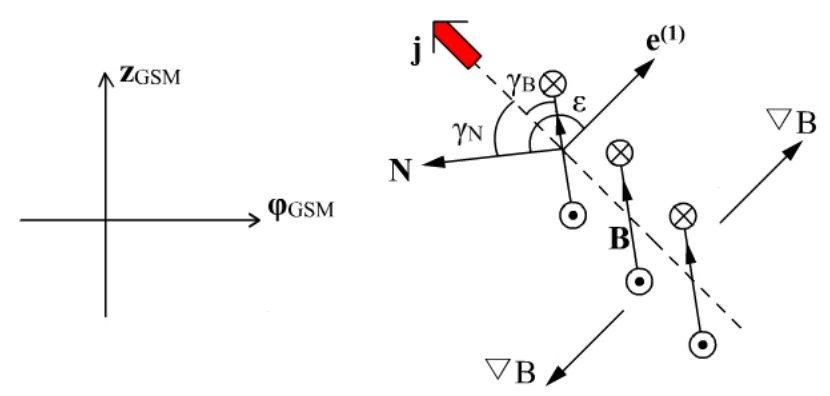

Fig. 2. Schematic illustration of the geometrical structure of the tilted current sheet during the Cluster crossing event on 5 August 2001 viewed toward the earth. The dashed line denotes the central plane of the current sheet. The red arrow represents the current density. The directions of the magnetic field in the MFLs have been marked in the figure. Several geometrical parameters of the current sheet are shown. $\hat{z}_{\mathrm{GSM}}$ and $\hat{\varphi}_{\mathrm{GSM}}$ are the base vectors along the $\mathrm{z}$ axis and azimuthal directions in GSM coordinates, respectively.

the normal of the current sheet $\left(\boldsymbol{e}^{(1)}\right)$ and the plane of the MFLs in the NS, that is the complementary angle of the angle $\varepsilon$ between $\boldsymbol{e}^{(1)}$ and the binormal $\hat{N}$ of the MFLs (see Fig. 2), i.e.,

$\delta \approx\left|90^{\circ}-\varepsilon\right|$,

the slipping angle (or tilt angle) $\delta$ can also be defined equivalently as the angle $\gamma_{N}$ between the current density vector $\left(\boldsymbol{j}=\mu_{0}^{-1} \nabla \times \boldsymbol{B}\right)$ and the binormal $\hat{\boldsymbol{N}}$ of the MFLs in the NS (see Fig. 2), i.e.,

$\delta \approx \gamma_{N}$.

When the slipping angle $\delta=0^{\circ}$, there is no tilting and it is a normal current sheet; if the slipping angle $\delta=90^{\circ}$, it is a vertical current sheet.

Panels 6-9 of Fig. 1b show the distribution of the current density in this thin current sheet. (Note that $\left[\hat{\boldsymbol{b}}, \boldsymbol{\rho}_{c} / \rho_{c}, \hat{\boldsymbol{N}}\right]$ constitute the local natural orthogonal coordinates on the MFLs (Shen et al., 2003, 2007a, 2008).) It is remarkable that for this tilted current sheet the current density has a bifurcated profile (Runov et al., 2003; Zelenyi et al., 2004; Asano et al., 2005; Nakamura et al., 2006; Thompson et al., 2006), and the two humps of the current density appear at about the two boundaries of the neutral sheet. It is also noted that the two humps of the current density are in correlated with the two peaks of the gradient of magnetic strength, $\nabla B$ (panel 7 of Fig. 1a). This may imply the contribution of the gradient drift motion of the particles. Within the neutral sheet, $j_{z}>0$ and $j_{B}>0$, so the current density has a positive $\mathrm{Z}$ component and a positive field-aligned component, implying the current sheet leans southward at the dawn side. Within the neutral sheet, the field-aligned component $j_{B}$ of the current density contributes a significant part of the total current density. The angle $\gamma_{B}$ of the current direction from the magnetic field direction decreases to $\gamma_{B} \approx 36.8^{\circ}\left(<90^{\circ}\right)$ at the center of the neutral sheet. The angle $\gamma_{N}$ between the current density and the binormal $\hat{N}$ is rather stable in the neutral sheet, and $\gamma_{N} \approx 54^{\circ}$ at the center. The angle $\varepsilon$ between the first $\boldsymbol{e}^{(1)}$ and the binormal $\hat{N}$ of the MFLs varies as Cluster crosses the current sheet, and its averaged value in the neutral sheet is $141^{\circ}$, which yields a slipping angle of about $\delta \approx\left|90^{\circ}-\varepsilon\right| \approx 51^{\circ}$.

The parameters of this tilted current sheet are summarized in Table 1. Figure 2 demonstrates the geometrical configuration of the tilted current sheet during the Cluster crossing event on 5 August 2001. In short, this is a tilted current sheet with a slipping angle of about $\delta \approx 54^{\circ}$, in which the current flows duskward and northward. The MFLs in this thin current sheet are plane curves which are about vertical to the equatorial plane. The normal of this current sheet is about $\left(44^{\circ}, 283^{\circ}\right)$. The half width of this tilted current sheet is $H \approx 0.8 R_{E}$, the half thickness of the neutral sheet is about $h \approx 0.19 R_{E}$, and the minimum curvature radius at the center of the NS is $R_{c, \min } \approx 0.31 R_{E}$.

\subsubsection{Case on 3 August 2004}

Figure $3 \mathrm{a}$ and $3 \mathrm{~b}$ show another tilted current sheet crossing event on 3 August 2004. Cluster is at $19.1 R_{E}$ geocentric distance, and its position angle in GSM is $\left(86.6^{\circ}, 211^{\circ}\right)$, so that this tilted CS appears lies near equatorial plane at a local time of about 02:00 LT. During this period, the AE index is about $150 \mathrm{nT}$ and the magnetic activity is quiet. The two boundaries of the neutral sheet are at 09:10:00 and 09:14:06 UT, respectively, as marked as two vertical black lines, and the center of the neutral sheet /current sheet is at 09:13:00 UT, denoted by a vertical red line, where the magnetic strength has a minimum value. 


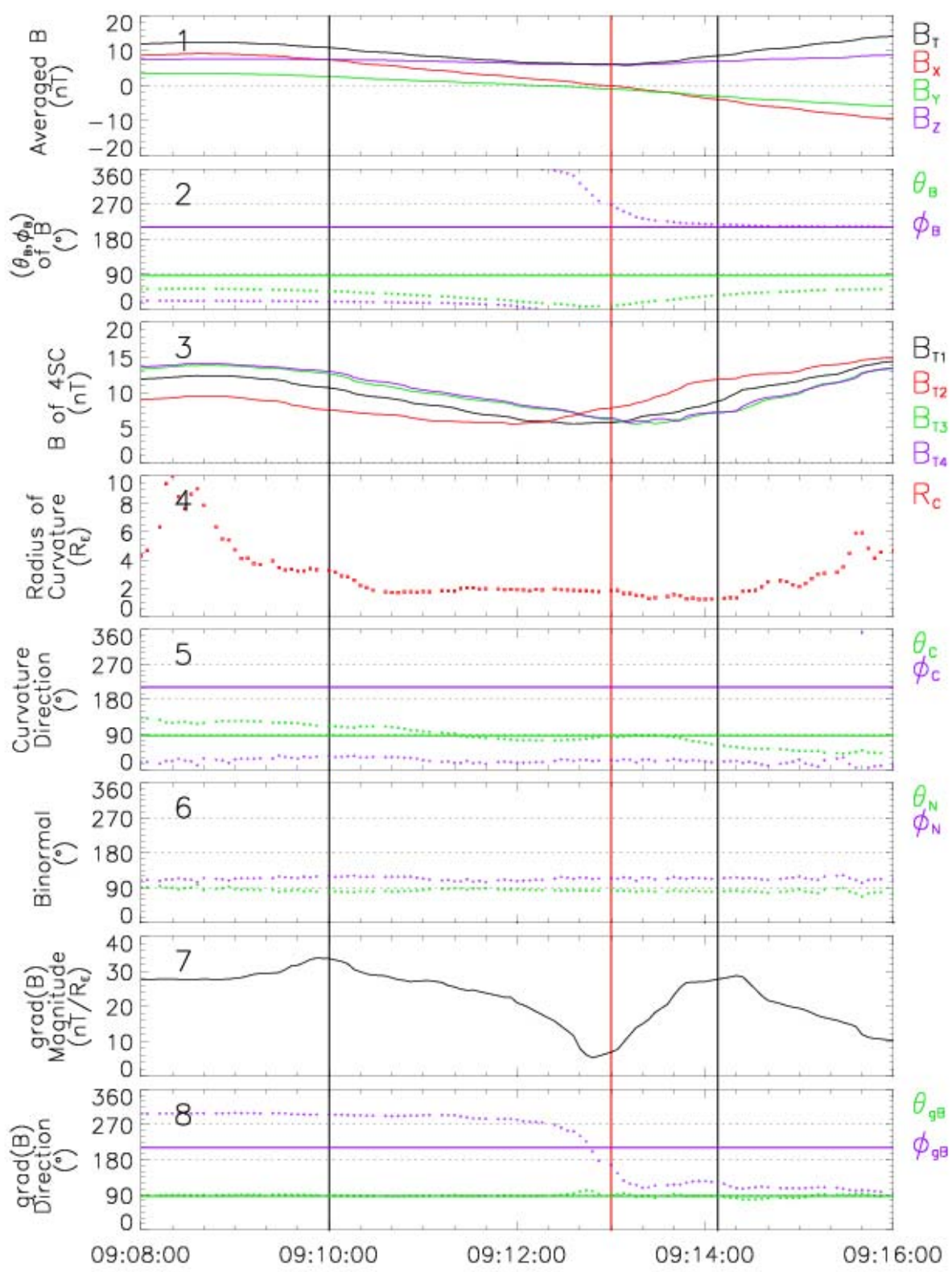

UT of the Day 20040803

Fig. 3a. The geometric structure of the tilted current sheet during one Cluster crossing event on 3 August 2004. The format of the figure is the same as Fig. 1a.

Panels 1-3 of Fig. 3a show the magnetic field in this current sheet. At the center of the current sheet, the magnetic field points northward and has a minimum value. Panels 4-6 of Fig. 3a show the configuration of magnetic field lines in this current sheet. As seen from panel 4 of Fig. 3a, the curvature radius of the MFLs in the neutral sheet is rather larger, around $2 R_{E}$. Panel 6 shows that the curvature points earthward, and that the binormal of the MFLs in the CS is more or less constant with a direction of $\left(\theta_{N}, \varphi_{N}\right) \approx\left(83^{\circ}, 114^{\circ}\right)$, indicating the MFLs in the current sheet are plane curves about vertical to the equatorial plane. Panels $7-8$ of Fig. 3a give the features of the gradient of the magnetic strength, $\nabla B$, which has a minimum value in the center of the CS, and reaches maximum values at the boundaries of the NS. The direction of $\nabla B$ points towards dawnside at the northern side $\left(B_{x}>0\right)$ and duskside at the southern side $\left(B_{x}<0\right)$ and it reverses its direction at the center of the CS. The direction angles $\left(\theta_{g B}, \varphi_{g B}\right)$ of $\nabla B$ are $\left(88.2^{\circ}, 296^{\circ}\right)$ at the northern side and $\left(87.4^{\circ}, 125^{\circ}\right)$ at the southern side, yielding the normal $\hat{\boldsymbol{n}}$ of the CS/NS as $\left(89.6^{\circ}, 111^{\circ}\right)$. Therefore, the normal $\hat{\boldsymbol{n}}$ of 


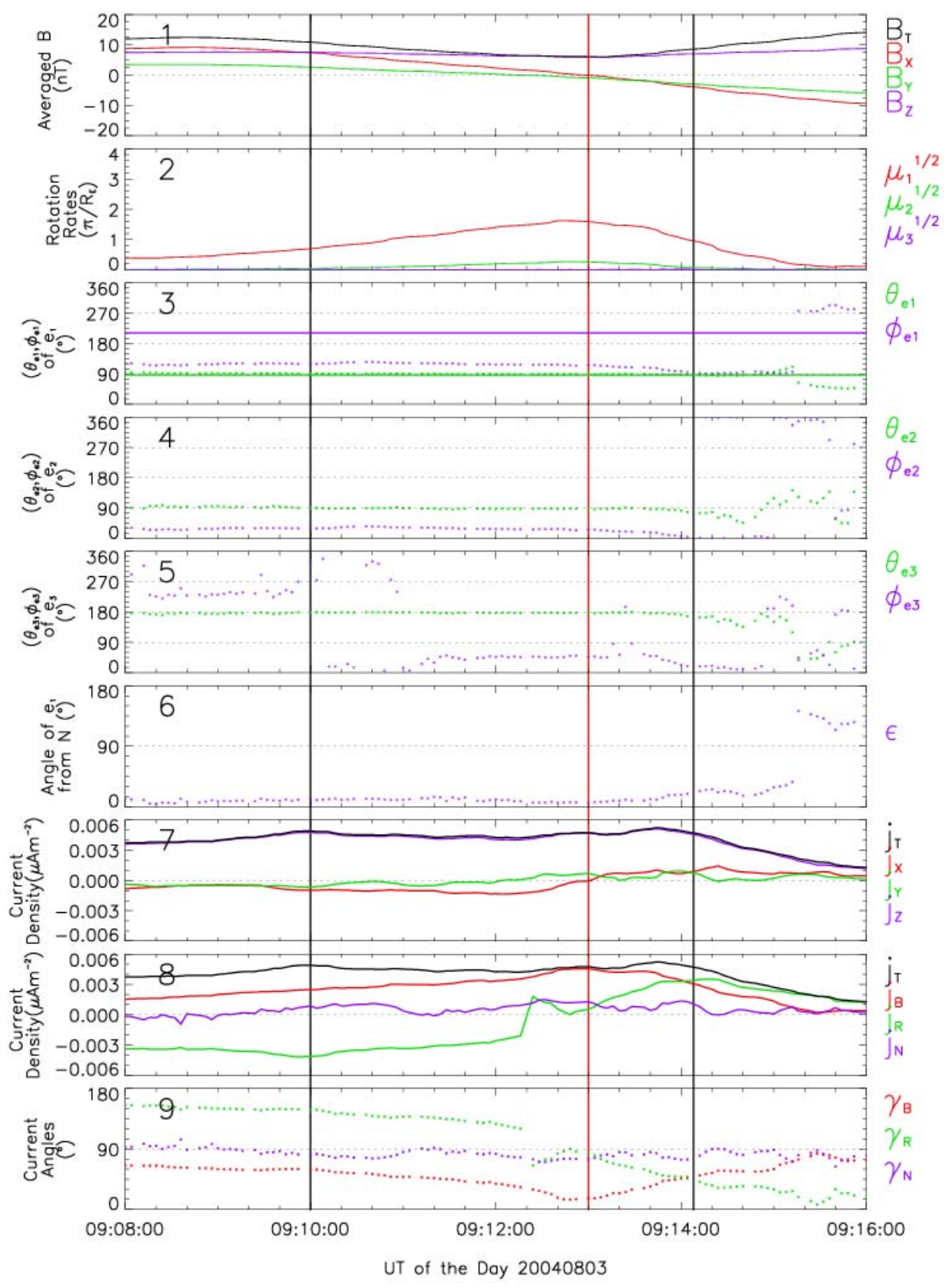

Fig. 3b. The geometric structure of the tilted current sheet during one Cluster crossing event on 3 August 2004. The format of the figure is the same as Fig. $1 b$.

the CS/NS is duskward and almost parallel to the binormal $\hat{N}$ of the MFLs. This CS is therefore nearly vertical to the equatorial plane, as demonstrated in Fig. 4.

Panels $2-5$ of Fig. $3 b$ illustrate the properties of the magnetic rotation in this current sheet event on 3 August 2004. The maximum magnetic rotation rate of the magnetic field vector $\mu_{1}^{1 / 2}$ is much larger than the medium rotation rate $\mu_{2}^{1 / 2}$, while the minimum rotation rate $\mu_{3}^{1 / 2}$ is nearly zero. Thus, this is a 1-D current sheet. The peak of the maximum magnetic rotation rate $\mu_{1}^{1 / 2}$ appears at the center of the neutral sheet, so the magnetic field vector rotates fastest at the center of the neutral sheet. The direction of $\boldsymbol{e}^{(1)}$ is constant in the NS and is about $\left(89^{\circ}, 115^{\circ}\right)$ at the center. Thus, the normal $\hat{\boldsymbol{n}}$ of the CS/NS is $\left(89.5^{\circ}, 117^{\circ}\right)$, which is in agreement with that reduced from the above calculation of $\nabla B /|\nabla B|$. The half-thickness of the neutral sheet is $h \approx 1 / \mu_{1 \max }^{1 / 2} \approx\left(1.6 \pi / R_{E}\right)^{-1} \approx 0.20 R_{E}$, indicating this vertical current sheet is very thin. In this NS, $B_{\min } \approx 7.7 \mathrm{nT}$, $\langle j\rangle \approx 0.0047 \mu \mathrm{A} / \mathrm{m}^{2}$, so that the half thickness of the NS can also be estimated by Ampere's Law from Eq. (3) as $h_{j} \approx B_{\min } /\left(\mu_{0}\langle j\rangle\right) \approx 1300 \mathrm{~km} \approx 0.20 R_{E}$, which is almost the 


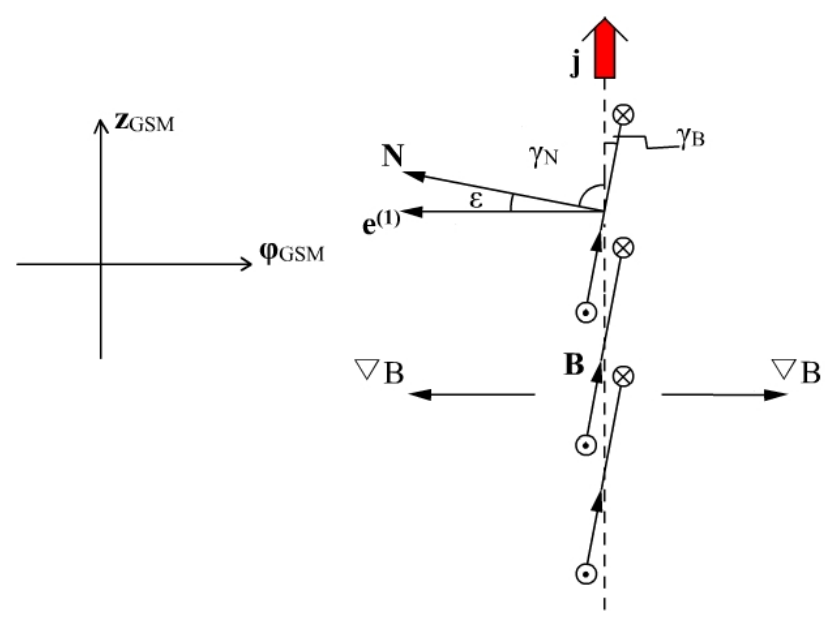

Fig. 4. Schematic illustration of the geometrical structure of the severely tilted current sheet during the Cluster crossing event on 3 August 2004 viewed toward the earth. The dashed line denotes the central plane of the current sheet. The red arrow represents the current density. The directions of the magnetic field in the MFLs have been marked in the figure. Several geometrical parameters of the current sheet are shown. $\hat{z}_{\mathrm{GSM}}$ and $\hat{\varphi}_{\mathrm{GSM}}$ are the base vectors along the $\mathrm{z}$ axis and azimuthal directions in GSM coordinates, respectively.

same as that determined from Eq. (2). The angle $\varepsilon$ between $\boldsymbol{e}^{(1)}$ and the binormal $\hat{N}$ is very small, and is approximately $6.7^{\circ}$ at the center of NS (panel 6). Therefore, the tilt angle of the current sheet can be yielded from Eq. (4) as $\delta \approx\left|90^{\circ}-\varepsilon\right| \approx 83.3^{\circ}$.

Panels 7-9 of Fig. 3b show the distribution of the current density in the current sheet. As illustrated in panel 7 of Fig. $3 b$, the northern component $j_{z}$ is positive and occupies nearly all the current density, so that the current in the current sheet is almost northward or in the $\mathrm{Z}$ direction, further indicating that this current sheet is vertical to the equatorial plane. Similar to the first tilted current sheet on 5 August 2001 investigated above, the current density has one bifurcated profile (Runov et al., 2003; Zelenyi et al., 2004; Asano et al., 2005; Nakamura et al., 2006; Thompson et al., 2006), the current density maximizes at the two boundaries of the NS and minimizes at the center of the neutral sheet. And also the two humps of the current density overlap the two peaks of the gradient of the magnetic strength. At the center of the neutral sheet, the current becomes almost field-aligned (panel 8), and the angle $\gamma_{B}$ of the current from the magnetic field is about $16^{\circ}$ at the center of the NS (panel 9). The angle $\gamma_{N}$ of the current from the binormal $\hat{N}$ remains constant and is approximately $75^{\circ}$ at the center of the NS.

The parameters of this tilted current sheet are listed in Table 1. Figure 4 shows the demonstration of the geometrical structure of the tilted current sheet during the Cluster crossing event at 09:08-09:16 UT on 3 August 2004. In short, this

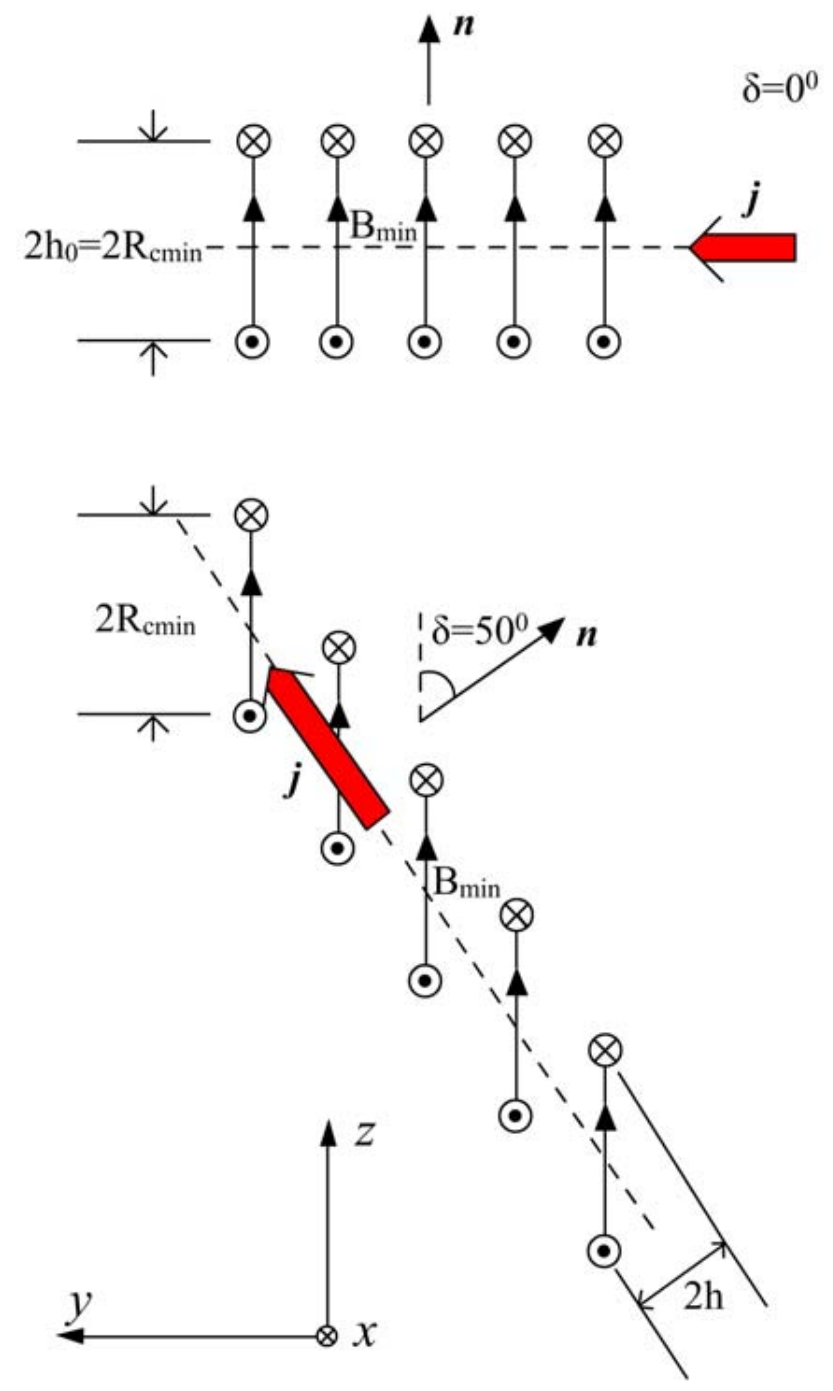

Fig. 5. The transformation from the normal Harris type current sheet (upper panel) to the tilted current sheet (lower panel) through the slipping of the MFLs.

current sheet is a vertical current sheet with a tilted angle of about $75^{\circ}$. The current in this tilted CS flows almost along $\mathrm{Z}$ direction and has a large field-aligned component at the center of its NS. Its normal is duskward at a direction of about $\left(89.6^{\circ}, 111^{\circ}\right)$.

We may check the relationship between the thickness of the NS and the tilt angle for one of these tilted current sheets. A Harris type current sheet with no guiding field $B_{y}$ is shown in the upper panel in Fig. 5. If the neighboring MFLs are slipping from each other while the configuration and orientation of the MFLs remain unchanged (see the lower panel in Fig. 5), the magnetic field (including its strength and direction) at any fixed point of one MFL will not vary, so that the two boundaries of the NS will not change their locations at the MFLs of the current sheet. 


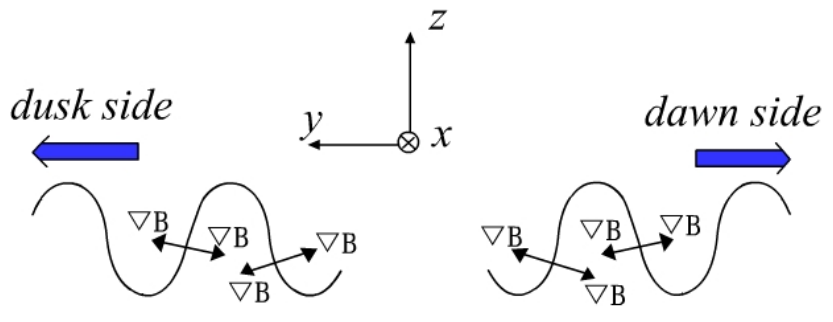

Fig. 6. Illustration of the propagation of the flapping waves of the tail tilted current sheet.

Therefore the thickness of the NS (that is the distance between the two boundaries of the NS) in the tilted current sheet is

$2 h \approx 2 h_{0} \cos \delta$.

For the Harris type current sheet with no guiding field By, the half-thickness $h_{0}$ of the NS is equal to the minimum radius of curvature, $R_{c, \min }$, of MFLs in the current sheet, i.e., $h_{0}=R_{c, \text { min }}$ (see Appendix A in Shen et al., 2007a). So that

$h \approx R_{c, \min } \cos \delta$.

The above formula relates the half-thickness $h$ of the NS to the minimum radius of curvature, $R_{c, \min }$, of MFLs and the tilt angle $\delta$ of the current sheet. The parameters for the two events in Table 1 satisfy this formula well. Equation (7) implies that the larger the tilt angle of the current sheet, the thinner its NS and the larger the current density in the neutral sheet (Eq. 3). Runov et al. (2005a) have argued that the thickness of the current sheet has no relationship with the minimum radius of curvature of MFLs because they have not included this influence of the tilt angle.

\subsection{Flapping motion propagation of the tail current sheet}

In Sect. 2.1, the features of the tail tilted current sheet have been investigated by analyzing Cluster 4 point magnetic observations. Typically, the tilted current sheet is closely related to the flapping motion of the tail current sheet. Here we may explore the properties of the tail current sheet flapping motion and its possible origin.

Here, by determining propagation directions based on the magnetic gradient, we may try a new perspective, so that one can determine the propagation direction of current sheet flapping waves easily and intuitively. (Obviously, the timing analysis (Russell et al., 1983; Harvey, 1998; Schwartz, 1998; Dunlop and Woodward, 1998; Sonnerup et al., 2008) can also be used to analyse the propagation direction and velocity of the current sheet flapping.) Figure 6 shows the sketched flapping waves of the tail current sheet, assumed to propagate towards flanks in the pre-midnight or post-midnight region. Generally, in the tail current sheet, the gradient of the magnetic strength $\nabla B$ points outward from the center of the current sheet. The measured $\nabla B$ will reverse its direction when one current sheet crosses the spacecraft. When the flapping waves propagate towards the dawnside (duskside), $\nabla B$ measured by the S/C will points first dawnward (duskward) with its azimuthal angle $\varphi_{g B}$ near to $270^{\circ}\left(90^{\circ}\right)$, and then duskward (dawnward) with its azimuthal angle $\varphi_{g B}$ near to $90^{\circ}\left(270^{\circ}\right)$. Therefore, the propagation directions of the flapping motion of the tail current sheet can be determined visually from the variation of the directional angle of $\nabla B$, as demonstrated in Fig. 6.

The second event introduced above in fact has a larger interval of interest in this regard. During 06:30-09:30 UT on 3 August 2004, Cluster observed one tail current sheet flapping event, which has also been studied previously by Petrukovich et al. (2006). When this CS flapping event occurs, Cluster is at $19 R_{E}$ geocentric distance, and its position angles in GSM coordinates are $(\theta, \varphi)=\left(84^{\circ}, 211^{\circ}\right)$ with a local time of $2.1 \mathrm{~h}$. Cluster is $9.64 R_{E}$ away from the midnight meridian plane at the dawnside tail. The average Cluster tetrahedron size is approximately $890 \mathrm{~km}$. Cluster is in fact situated close to apogee and hardly moves at all in GSM in the three hours indicated.

Figure $7 \mathrm{a}$ and $\mathrm{b}$ demonstrates the variations of the various parameters of the magnetic field as Cluster crosses the CS periodically during this flapping event, in which the centers of the NS are marked by red vertical lines. Each time Cluster crosses the center of the NS, the magnetic strength drops to the minimum and the Bx changes its sign (panel 1 of Fig. 7a). The polar angle $\theta_{B}$ becomes very small (panel 2 of Fig. 7a), implying the magnetic field is nearly northward there. The curvature radius of the MFLs minimizes (panel 4 of Fig. 7a) and the gradient of the magnetic strength has the lowest value (panel 7 of Fig. 7a) and reverses its direction (panel 8 of Fig. 7a). The magnetic rotation rates peak at the center of the NS (panel 2 of Fig. 7b) and the current density has very significant $\mathrm{Z}$ component $j_{z}$ and field-aligned component $j_{B}$. The parameters at the center of the NS during these series of CS crossing events are listed in Table 2. As shown in panel 6 of Fig. 7a, during this CS flapping event, the binormal $\hat{N}$ of the MFLs is nearly constant and is about $\left(80^{\circ}, 120^{\circ}\right)$ on average. The normal $\hat{\boldsymbol{n}}$ of the NS, as determined by the first eigenvector $\hat{\boldsymbol{e}}^{(1)}$ in Table 2, deviated considerably from the northern direction, and generally leans to the flank sides. Therefore, from the features of the NS deduced here, we may believe that the current sheet is tilted as its NS crosses Cluster and that the tail current sheet is flapping. The various parameters for each tilted current sheet at the Cluster crossing times are listed in Table 2. It is noted that the half thickness $h$ of the NS, the tilt angle $\delta$ and the minimum radius of curvature, $R_{c, \min }$, of MFLs in the current sheet, as shown in Table 2, basically obey the relationship $h \approx R_{c, \min } \cos \delta$ (Eq. 7). With temporal evolution of the flapping motion, the slipping angle $\delta$ of the tail current sheet generally gets larger and larger, while the half thickness $h$ of the NS becomes smaller and smaller and the current density in the NS grows stronger and stronger. At the end, the tilted 


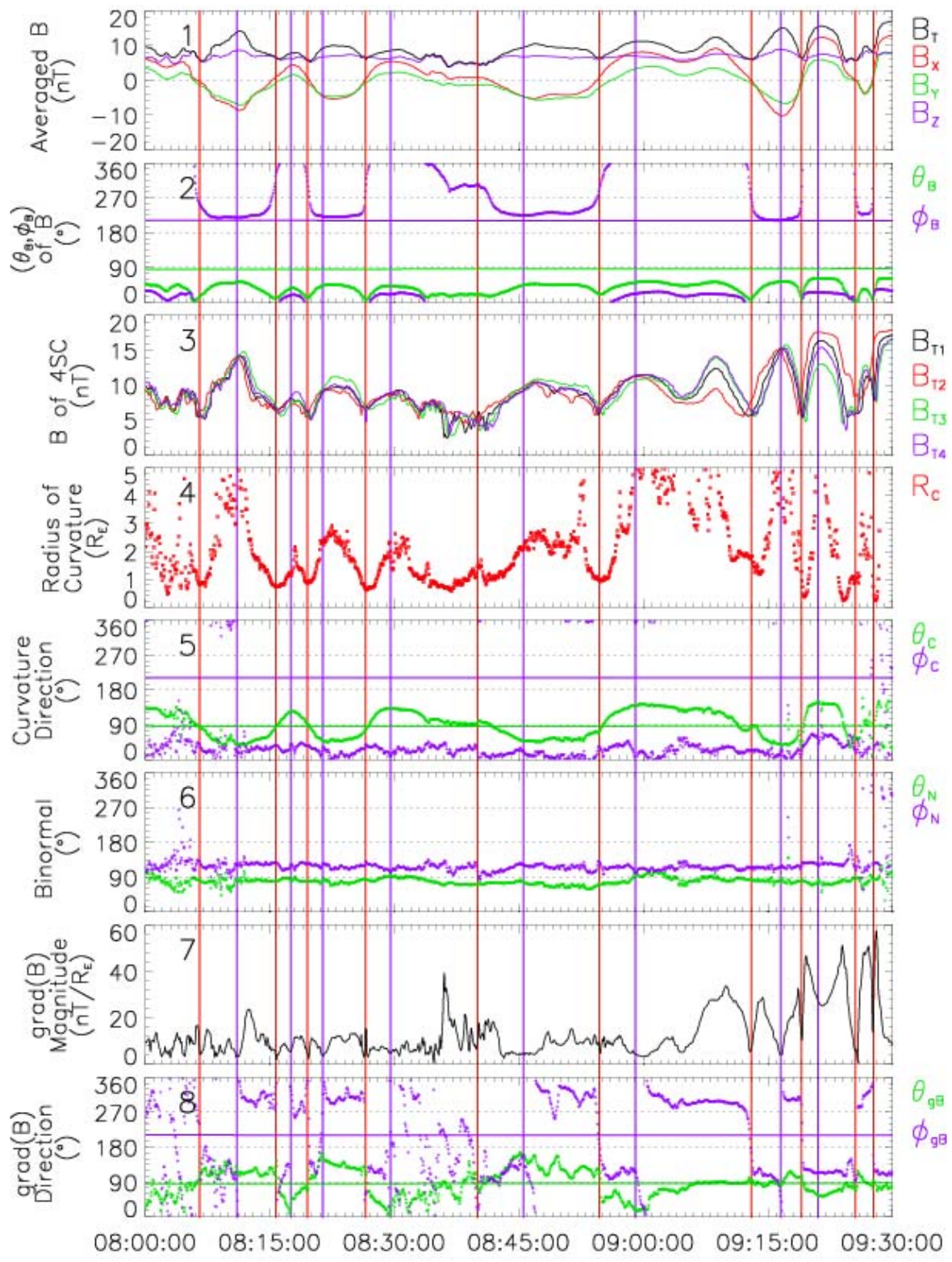

UT of the Day 20040803

Fig. 7a. The geometric structure of the tilted current sheet during a series of Cluster crossings on 3 August 2004. The format of the figure is just same as that of Fig. 1a.

current sheet becomes a very-nearly vertical sheet. These features manifest the gradually growing of the tail flapping motion during 08:00-09:30 UT.

Between the neighboring NS crossing events, Cluster lies at regions where the current sheet becomes a normal one, as marked by violet vertical lines in Fig. 7a and b. During these special periods, the flapping wave motion is sampled at its wave crests or wave troughs. At these times, the first eigenvector $\hat{\boldsymbol{e}}^{(1)}$ (panel 3 of Fig. 7b), may be regarded as the normal $\hat{\boldsymbol{n}}$ of the NS and is about northward. The angle $\varepsilon$ is about $90^{\circ}$ (panel 6 of Fig. 7b), thus the tilt angle $\delta$ is nearly $0^{\circ}$ and the current density is almost totally duskward (panel 7 of Fig. 7b) and $\gamma_{N}$ is nearly always zero (panel 9 of Fig. 7b).

During each NS crossing, the direction of $\nabla B$ is first dawnward with its azimuthal angle $\varphi_{g B}$ near to $270^{\circ}$, and then duskward with its azimuthal angle $\varphi_{g B}$ near to $90^{\circ}$, so that all the tilted current sheets measured are moving toward the dawnside and there are flapping waves in the tail current sheet propagating dawnward. The normal velocity of each NS is listed in Table 2 and we may estimate the phase velocity of the flapping waves. During 08:00-09:30 UT, the averaged tilted angle is $\langle\delta\rangle \approx 67^{\circ}$, the averaged normal velocity 


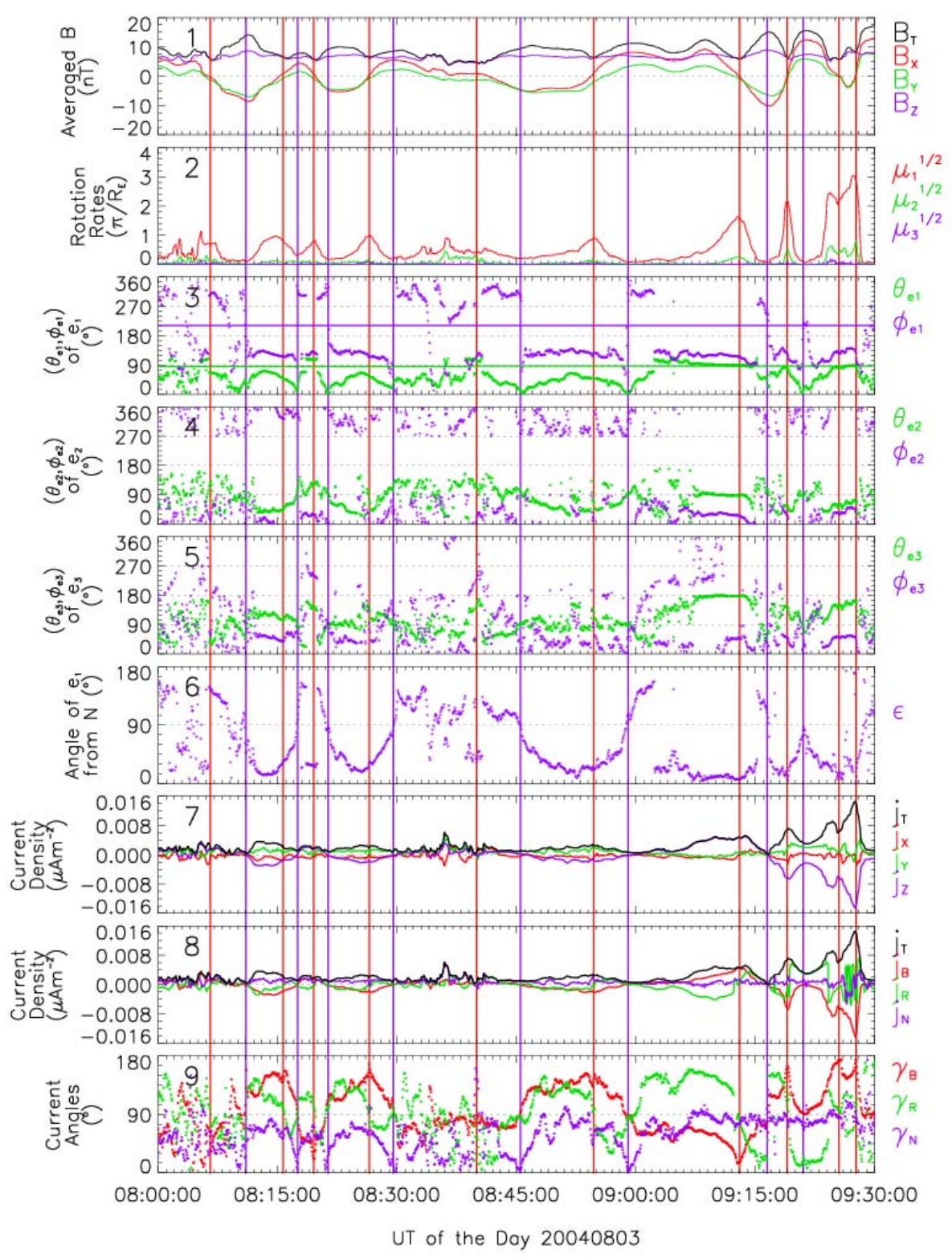

Fig. 7b. The geometric structure of the tilted current sheet during a series of Cluster crossings on 3 August 2004. The format of the figure is the same as Fig. $1 b$.

of the NS is $\left\langle V_{n}\right\rangle \approx 27 \mathrm{Km} / \mathrm{s}$. Thus, the phase velocity of the flapping waves is $\left\langle V_{P}\right\rangle \approx\left\langle V_{n}\right\rangle / \sin \langle\delta\rangle \approx 29 \mathrm{Km} / \mathrm{s}$. The averaged period of the flapping waves is $\langle T\rangle \approx 14 \mathrm{~min} \mathrm{~s}$, so the expected wavelength is $\langle\lambda\rangle \approx\left\langle V_{P}\right\rangle \cdot\langle T\rangle \approx 3.8 R_{E}$. Based on the above analysis, we may give a demonstration of the structure of ideal tail current sheet flapping waves propagating dawnward as in Fig. 8.

In order to make clear the features of the tail current sheet flapping waves, we need to investigate more events. Table 3 shows the deduced features of the six important tail current sheet flapping events from our methods (some physical parameters are not available for the event on 26 September
2001 because Cluster is outside of the neutral sheet), which have also been investigated in the literature (Sergeev et al., 2003, 2004; Runov et al., 2005a, b; Zhang et al., 2002, 2005; Petrukovich et al., 2006). The locations of all these events are about $10 R_{E}$ away from the midnight meridian and much nearer to the dawnside or duskside flanks. For these tail flapping events, the minimum curvature radius of the MFLs in the current sheet is rather large, with typical values of $0.32 \sim 1.5 R_{E}$, while the neutral sheet, as Cluster crosses, is very thin, with the averaged half thickness being about $0.14 \sim 0.36 R_{E}$. This is caused by the slipping of the MFLs and governed approximately by Eq. (7). While the 
Table 2. The parameters of the magnetic field at the center of the neutral sheet during the series of CS crossing events on 3 August 2004.

\begin{tabular}{cccccccccccc}
\hline Time & $\begin{array}{c}R_{c, \min } \\
\left(R_{E}\right)\end{array}$ & $\begin{array}{c}\hat{\boldsymbol{e}}^{(1)}(\hat{\boldsymbol{n}}) \\
\left(\theta_{e 1}, \varphi_{e 1}\right)\end{array}$ & $\begin{array}{c}\hat{\boldsymbol{b}} \\
\left(\theta_{B}, \varphi_{B}\right)\end{array}$ & $\begin{array}{c}\mu_{1 \max }^{1 / 2} \\
\left(\pi / R_{E}\right)\end{array}$ & $\begin{array}{c}j \\
\left(\mu \mathrm{A} / \mathrm{m}^{2}\right)\end{array}$ & $\gamma_{B}$ & $\gamma_{N}$ & $\delta \approx\left|90^{\circ}-\varepsilon\right|$ & $\begin{array}{c}\hat{\boldsymbol{N}} \\
\left(\theta_{N}, \varphi_{N}\right)\end{array}$ & $\begin{array}{c}h \\
\left(R_{E}\right)\end{array}$ & $\begin{array}{c}V_{n} \\
(\mathrm{~km} / \mathrm{s})\end{array}$ \\
\hline $08: 06: 30$ & 0.83 & $\left(64^{\circ}, 306^{\circ}\right)$ & $\left(16^{\circ}, 253^{\circ}\right)$ & 0.69 & 0.0016 & $39^{\circ}$ & $53^{\circ}$ & $54^{\circ}$ & $\left(80^{\circ}, 126^{\circ}\right)$ & 0.46 & 31 \\
$08: 15: 40$ & 0.74 & $\left(48^{\circ}, 116^{\circ}\right)$ & $\left(11^{\circ}, 322^{\circ}\right)$ & 0.80 & 0.0022 & $144^{\circ}$ & $56^{\circ}$ & $57^{\circ}$ & $\left(80^{\circ}, 127^{\circ}\right)$ & 0.40 & 13 \\
$08: 19: 30$ & 0.88 & $\left(106^{\circ}, 129^{\circ}\right)$ & $\left(12^{\circ}, 307^{\circ}\right)$ & 0.80 & 0.0017 & $26^{\circ}$ & $68^{\circ}$ & $63^{\circ}$ & $\left(79^{\circ}, 125^{\circ}\right)$ & 0.40 & 27 \\
$08: 26: 30$ & 0.69 & $\left(55^{\circ}, 115^{\circ}\right)$ & $\left(6.9^{\circ}, 288^{\circ}\right)$ & 0.95 & 0.0019 & $161^{\circ}$ & $78^{\circ}$ & $61^{\circ}$ & $\left(84^{\circ}, 126^{\circ}\right)$ & 0.34 & 19 \\
$08: 40: 00$ & 1.4 & $\left(104^{\circ}, 115^{\circ}\right)$ & $\left(20^{\circ}, 304^{\circ}\right)$ & 0.39 & 0.00067 & $77^{\circ}$ & $154^{\circ}$ & $54^{\circ}$ & $\left(71^{\circ}, 105^{\circ}\right)$ & 0.82 & 49 \\
$08: 54: 40$ & 0.97 & $\left(49^{\circ}, 125^{\circ}\right)$ & $\left(20^{\circ}, 293^{\circ}\right)$ & 0.88 & 0.0025 & $140^{\circ}$ & $64^{\circ}$ & $67^{\circ}$ & $\left(70^{\circ}, 122^{\circ}\right)$ & 0.36 & 24 \\
$09: 13: 00$ & 1.8 & $\left(89^{\circ}, 115^{\circ}\right)$ & $\left(8.7^{\circ}, 269^{\circ}\right)$ & 1.6 & 0.0047 & $16^{\circ}$ & $75^{\circ}$ & $83.3^{\circ}$ & $\left(82^{\circ}, 114^{\circ}\right)$ & 0.20 & 9.2 \\
$09: 19: 00$ & 1.3 & $\left(79^{\circ}, 125^{\circ}\right)$ & $\left(12^{\circ}, 267^{\circ}\right)$ & 2.1 & 0.0068 & $162^{\circ}$ & $81^{\circ}$ & $80^{\circ}$ & $\left(81^{\circ}, 116^{\circ}\right)$ & 0.15 & 40 \\
$09: 25: 15$ & 0.96 & $\left(82^{\circ}, 127^{\circ}\right)$ & $\left(2.1^{\circ}, 298^{\circ}\right)$ & 2.2 & 0.0062 & $172^{\circ}$ & $86^{\circ}$ & $75^{\circ}$ & $\left(88^{\circ}, 114^{\circ}\right)$ & 0.14 & 17 \\
$09: 27: 40$ & 0.77 & $\left(83^{\circ}, 113^{\circ}\right)$ & $\left(12^{\circ}, 280^{\circ}\right)$ & 2.8 & 0.014 & $169^{\circ}$ & $87^{\circ}$ & $78^{\circ}$ & $\left(85^{\circ}, 123^{\circ}\right)$ & 0.11 & 17 \\
\hline
\end{tabular}

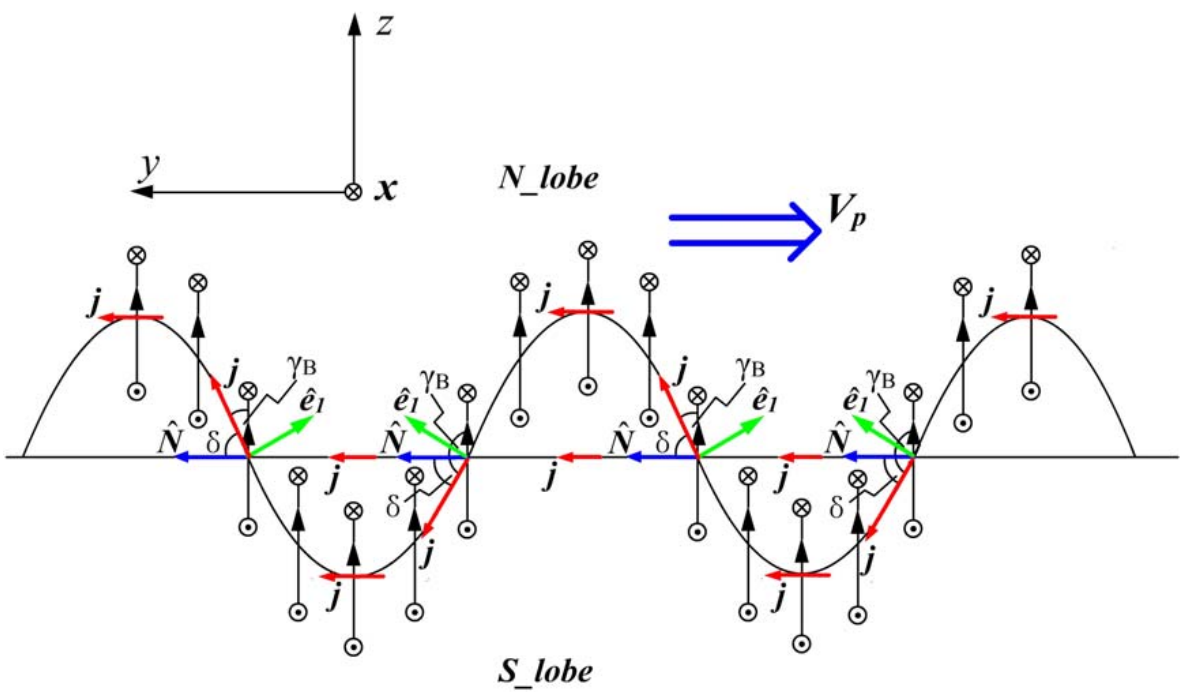

Fig. 8. Schematic illustration of the ideal flapping waves moving dawnward in the tail.

flapping waves are passing by Cluster, the current sheet is tilted severely, with the tilt angle being about $65^{\circ} \sim 75^{\circ}$. The phase velocities of these flapping waves are approximately $22 \sim 72 \mathrm{Km} / \mathrm{s}$, while their periods and wavelengths are about $10 \sim 32 \mathrm{~min}$ and 3.8 8.1 $R_{E}$, respectively. As pointed out by other researchers (Sergeev et al., 2003, 2004; Runov et al., 2005a, b; Zhang et al., 2002, 2005; Petrukovich et al., 2006), the flapping waves tend to propagate outward from the midnight meridian, i.e., the flapping waves move dawnward at the dawn side and duskward at the dusk side. The flapping motions usually last several hours, as shown in Table 3, indicating that there is a continuous and stable energy supply.

With Cluster and DSP (Liu et al., 2005) conjunction tail observations, Zhang et al. (2005) have found that the spatial scale of the flapping motions along the tail direction is larger than $5 R_{E}$ and they may occur at tailward distance of
$11 \sim 30 R_{E}$. Previous analysis (Sergeev et al., 2003, 2004, 2006; Zhang et al., 2002, 2005) has argued that the sources of the flapping motion of tail current sheet is at the tail midnight meridian, possibly caused by bursty bulk flows (BBF) (Angelopoulos et al., 1994) during local energy transient dissipations (Sergeev et al., 1996). However, the transient energy release events in the near-earth midnight plasma sheet generally have a spatial scale of several $R_{E}$, and temporal scale of several minutes (Sergeev et al., 1996), while the tail flapping motions span a rather large part of the magnetotail (maybe the whole near earth magnetotail), lasting about several hours generally. And one fact is that during the six tail CS flapping events listed in Table 3, the magnetosphere is quiet or only has weak activities in general (see Table 3). Therefore, it is doubtful if the local transient energy release events in the near-earth midnight plasma sheet are able to 


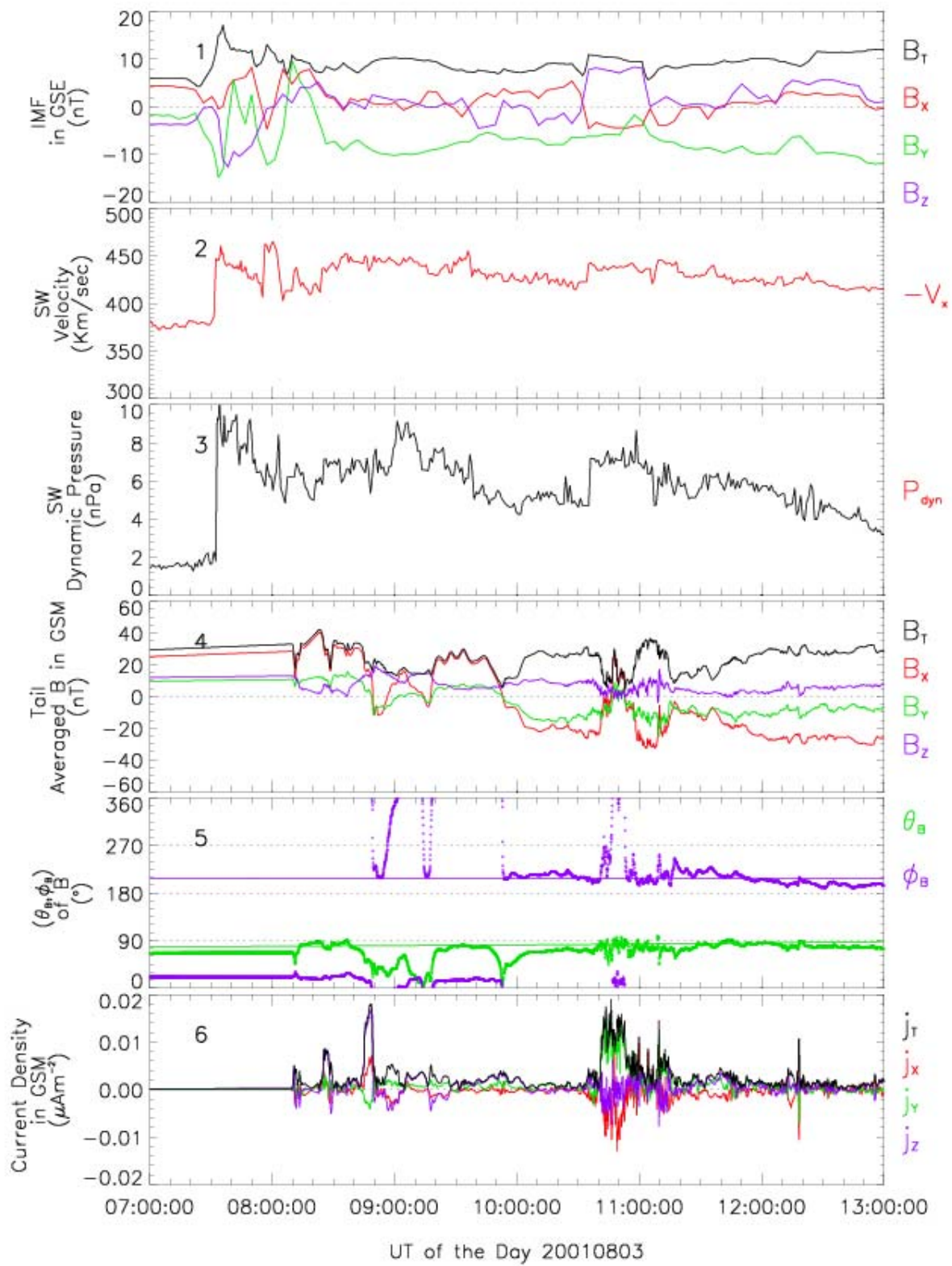

Fig. 9. The solar wind conditions as observed by the ACE spacecraft and the physical quantities of the tail current sheet measured by Cluster during the tail flapping event on 3 August 2001. Cluster is at $19 R_{E}$ geocentric distance, at the nightside equatorial plane, and its polar angle $\theta$ and azimuthal angle $\varphi$ in GSM are about $81^{\circ}$ and $207^{\circ}$, respectively. Panel 1: the interplanetary magnetic field (IMF) in GSE; panel 2: The velocity of the solar wind; panel 3: the dynamic pressure of the solar wind; panel 4: the magnetic field at the center of Cluster tetrahedron as are the average of those at the four S/C in GSM; panel 5: the directional angle $\left(\theta_{B}, \varphi_{B}\right)$ of the magnetic field at the center of Cluster tetrahedron in GSM; panel 6: The three components $\left(j_{x}, j_{y}, j_{z}\right)$ of the current density at the center of Cluster tetrahedron at GSM coordinates. In panels 4-6, the data before 08:10 UT are pseudo due to the unavailability of actual observation data.

supply the energy for sustaining the tail flapping motions with large temporal and spatial scales.

Based on the facts revealed here, we would suggest that the tail current sheet flapping motion is possibly the manifestation of the global oscillating motion of the tail caused by the interaction between the tail and the solar wind, the energy for which is provided by the solar wind. Figure 9 illustrates the tail flapping as observed by Cluster and solar wind conditions as observed by ACE satellite on 3 August 2001. The parameters of the solar wind have already been shifted from the L1 point to Earth's magnetosphere assuming the solar wind is moving at constant velocity. It can be seen from Fig. 9 that, a strong interplanetary shock has just impacted on the magnetosphere as the tail flapping motion occurs. Therefore, 


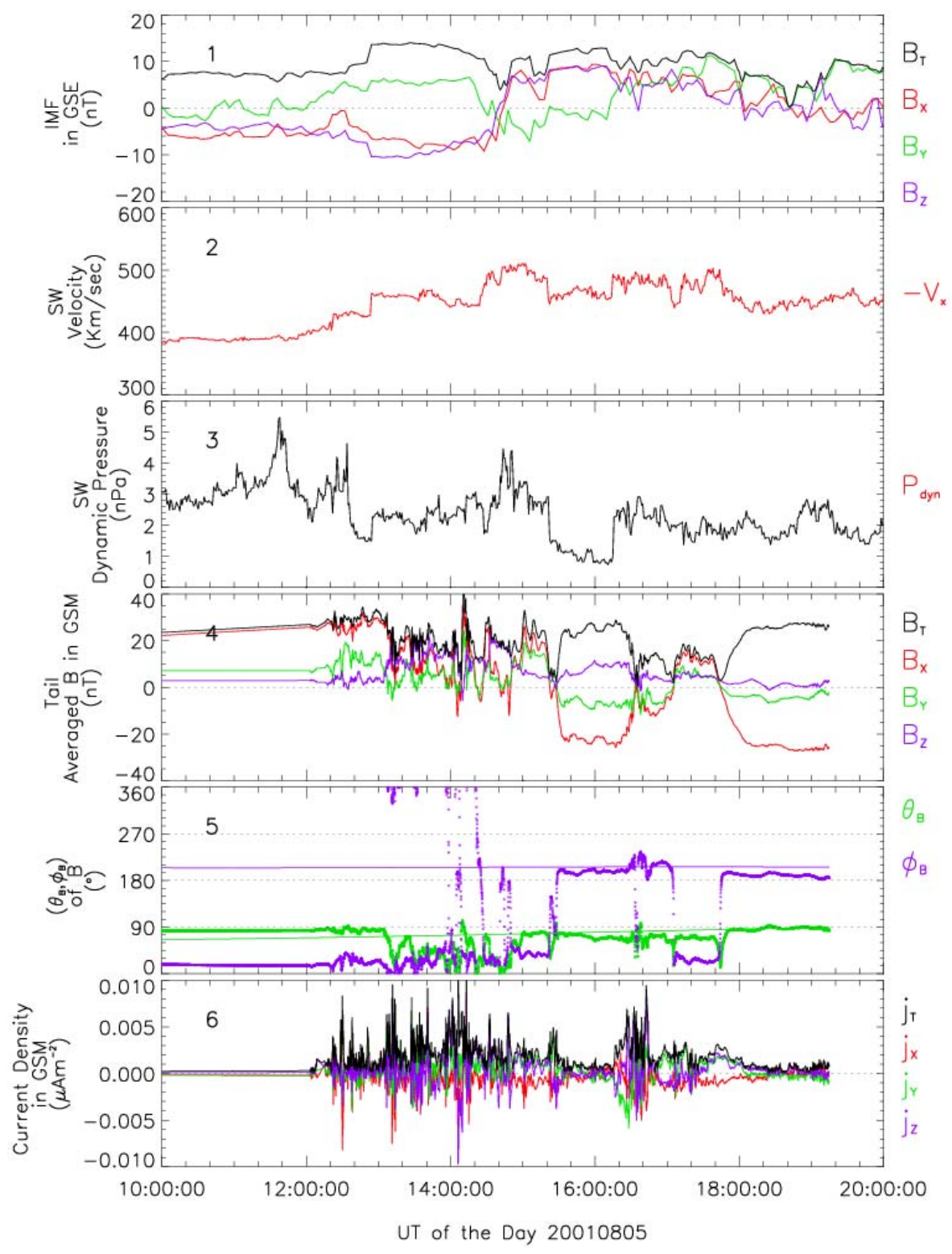

Fig. 10. The solar wind conditions as observed by ACE spacecraft and physical quantities of the tail current sheet measured by Cluster during the tail flapping event on 5 August 2001. Cluster is at $19.3 R_{E}$ geocentric distance, at the nightside equatorial plane, and its polar angle $\theta$ and azimuthal angle $\varphi$ in GSM are about $85^{\circ}$ and $206^{\circ}$, respectively. The format of the figure is just same as that of Fig. 11 . In panel $4-6$, the data before 12:00 UT are pseudo due to the unavailability of actual observation data.

the tail current sheet flapping motion on 3 August 2001 is possibly created by interaction between the magnetosphere and the solar wind with fast velocity and high dynamic pressure. Figure 10 demonstrates that another tail current sheet flapping event occurring on 5 August 2001. From Fig. 10 we may see that the tail flapping motion takes place during the solar wind velocity enhancement period. The current sheet flapping motion during 13:00-15:40 UT occurs within the interval of the recovery phase of a strong substorm; while the current sheet flapping motion during 15:40-18:00 UT appears when the magnetosphere is quiet. So that, the enhance- ment of the solar wind velocity is possibly the cause generating this tail flapping wave motion on 5 August 2001.

Therefore, the enhancements of the velocity or/and the dynamic pressure of the solar wind may possibly lead to the occurrence of the tail flapping motion. Figure 11 shows schematically the possible mechanism of the tail flapping motion. The tail flapping waves may be the standing waves of the tail propagating crossing the tail caused by the impact of the disturbed solar wind. More work will be required to make clear the mechanism of tail flapping. 


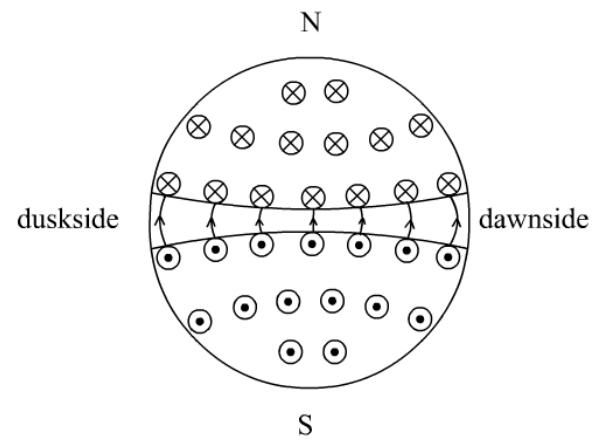

Steady tail

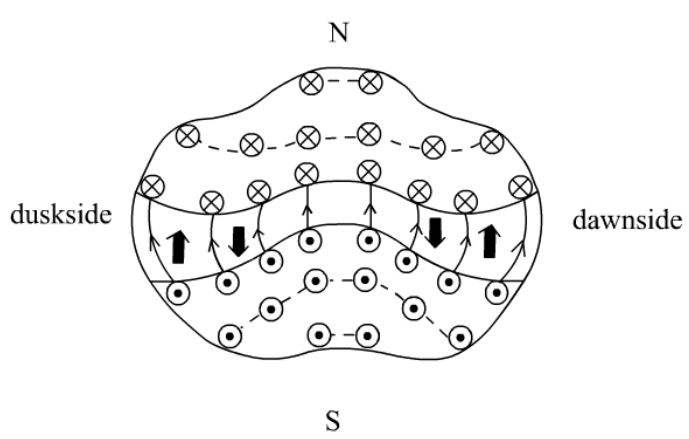

Flapping tail

Fig. 11. Demonstration of the flapping motion of the near earth tail caused by the impact of disturbed solar wind.

Table 3. Parameters of tail CS flapping events.

\begin{tabular}{|c|c|c|c|c|c|c|c|c|c|c|c|c|c|c|c|}
\hline Events & $\begin{array}{c}R \\
\left(R_{E}\right)\end{array}$ & $\begin{array}{c}(\theta, \varphi) \\
\text { in GSM }\end{array}$ & $\begin{array}{l}\text { LT } \\
(\mathrm{h})\end{array}$ & $\begin{array}{l}\mathrm{Y} \text { in GSM } \\
\quad\left(R_{E}\right)\end{array}$ & $\begin{array}{c}\left\langle B_{\min }\right\rangle \\
(\mathrm{nT})\end{array}$ & $\begin{array}{l}\left\langle R_{c, \min }\right\rangle \\
\quad\left(R_{E}\right)\end{array}$ & $\begin{array}{c}\langle h\rangle \\
\left(R_{E}\right)\end{array}$ & $\langle\delta\rangle$ & $\begin{array}{c}\left\langle V_{n}\right\rangle \\
(\mathrm{Km} / \mathrm{s})\end{array}$ & $\begin{array}{c}\left\langle V_{P}\right\rangle \\
(\mathrm{Km} / \mathrm{s})\end{array}$ & $\begin{array}{c}\langle T\rangle \\
(\min \mathrm{s})\end{array}$ & $\begin{array}{c}\langle\lambda\rangle \\
\left(R_{E}\right)\end{array}$ & Lifetime & $\begin{array}{l}\text { Propagation } \\
\text { direction }\end{array}$ & $\begin{array}{l}\text { Magnetospheric } \\
\text { activities }\end{array}$ \\
\hline $\begin{array}{l}3 \text { Aug 2001, } \\
08: 30-10: 30 \text { UT }\end{array}$ & 19 & $\left(81^{\circ}, 207^{\circ}\right)$ & 1.8 & -8.5 & 13 & 1.5 & 0.36 & $75^{\circ}$ & 21 & 22 & 32 & 6.6 & $2 \mathrm{~h}$ & dawnward & Later recovery \\
\hline $\begin{array}{l}5 \text { Aug 2001, } \\
\text { 13:00-18:00 U1 }\end{array}$ & $\Gamma^{19.3}$ & $\left(85^{\circ}, 206^{\circ}\right)$ & 1.7 & -8.4 & 12.0 & 0.6 & 0.24 & $58^{\circ}$ & 61 & 72 & 12 & 8.1 & $5 \mathrm{~h}$ & dawnward & $\begin{array}{l}\text { 13:00-15:40 UT: Strong; } \\
\text { 15:40-18:00 UT: quiet }\end{array}$ \\
\hline $\begin{array}{l}26 \text { Sep 2001, } \\
22: 15->23: 20\end{array}$ & $\begin{array}{l}19.3 \\
\text { UT }\end{array}$ & $\left(91^{\circ}, 158^{\circ}\right)$ & 22.5 & 7.2 & & & & & & & & & $>1 \mathrm{~h}$ & duskward & weak \\
\hline $\begin{array}{l}20 \text { Oct 2001, } \\
\text { 09:30-10:00 U1 }\end{array}$ & $T^{18}$ & $\left(94^{\circ}, 138^{\circ}\right)$ & 20.8 & 12 & 2.8 & 0.32 & 0.14 & $65^{\circ}$ & 40 & 44 & 10 & 4.2 & $0.5 \mathrm{~h}$ & duskward & quiet \\
\hline $\begin{array}{l}3 \text { Aug 2004, } \\
\text { 06:30-09:30 U1 }\end{array}$ & 19 & $\left(84^{\circ}, 211^{\circ}\right)$ & 2.1 & -9.8 & 6.3 & 1.0 & 0.28 & $67^{\circ}$ & 27 & 29 & 14 & 3.8 & $3 \mathrm{~h}$ & dawnward & quiet \\
\hline $\begin{array}{l}5 \text { Aug 2004, } \\
\text { 13:00-18:00 UT }\end{array}$ & $T^{18.5}$ & $\left(82^{\circ}, 210^{\circ}\right)$ & 2.0 & -9.2 & 6.2 & 0.95 & 0.28 & $65^{\circ}$ & 31 & 34 & 17.5 & 5.6 & $5 \mathrm{hrs}$ & dawnward & weak \\
\hline
\end{tabular}

\section{Summary and conclusions}

Tilted current sheets and current sheet flapping waves in the Earth's magnetotail are frequently occurring phenomena which have drawn much recent attention. The purpose of this paper is to clearly understand the geometrical structure of the tail tilted current sheet and current sheet flapping waves based on Cluster (Escoubet et al., 2001) multiple spacecraft measurements. To achieve this goal, previously developed multi-point data analysis methods (Shen et al., 2003, 2007a, b), i.e. curvature analysis and magnetic rotation analysis (MRA), are applied in this research. By using the previously developed multiple point data analysis methods (Shen et al., 2003, 2007a, b, 2008), we have investigated the geometrical structures of tilted current sheet and tail flapping waves. The configurations of the tilted current sheet have been directly and clearly revealed for the first time, as illustrated in Figs. 2, 4 and 5. As in previous investigations (Shen et al., 2007a), the neutral sheet (NS) is defined as the region within which $B \leq \sqrt{2} B_{\min }$ in the current sheet. The magnetic field vector rotates about $90^{\circ}$ in the NS (Shen et al., 2008b), so the NS is actually the magnetic vector rotation layer in the current sheet. We have given the explicit and quantitative definition of the tilt angle of the tilted current sheet for the first time: the tilt angle $\delta$ of the tilted current sheet as the complementary angle of the angle between the normal $\hat{\boldsymbol{n}}$ of the CS and the binormal $\hat{N}$ of the MFLs in the NS.

We summarize the common features of the tilted current sheet as follows:

1. The MFLs of the tilted CS are plane curves similar that of the normal CS except that the neighboring MFLs slip relative to each other in the plane of curvature. The osculating planes of the MFLs in the tilted CS are approximately vertical to the equatorial plane, and the binormals of the MFLs are generally along the dawn-dusk direction. However, the normal of the tilted CS deviates from the south-north $(Z)$ direction and leans to the flank sides.

2. The magnetic vector rotates substantially within the NS of the tilted current sheet. The half thickness of the NS is generally much less than the minimum radius of the curvature of the MFLs. The half thickness $h$ of the NS, the tilt angle $\delta$ and the minimum radius of curvature, $R_{c, \text { min }}$, of MFLs in the current sheet normally obey the 
relationship $h \approx R_{c, \text { min }} \cos \delta$. In general, the more tilted the current sheet, the thinner the NS.

3. In the NS of the tilted current sheet, the field-aligned current component is very large, has a one-peak profile and maximizes at the center of the CS.

4. The larger the tilt-angle of the tilted CS, the stronger the current density in its NS. In some of the tilted CS cases, the current density profile may have a bifurcated profile. The current density maximizes near the two boundaries of the NS and minimizes at the center of the neutral sheet, the two humps of the current density often superpose the two peaks of the gradient of magnetic strength. This may indicate the significant contribution of the magnetic gradient drift current.

The tilted current sheet typically appears along with the occurrence of tail current sheet flapping waves. Six current sheet flapping motion events have been analyzed in this research. It is found that, for these tail flapping events, the minimum radius of the curvature of the MFLs in the current sheet is about $0.32 \sim 1.5 R_{E}$ on average, while the averaged half thickness of the neutral sheet is only about $0.14 \sim 0.36 R_{E}$. During these tail flapping motion as measured by Cluster, the current sheet is tilted severely, its tilt angle may be about $65^{\circ} \sim 75^{\circ}$ in average. The phase velocities of these flapping waves are approximately $22 \sim 72 \mathrm{Km} / \mathrm{s}$, and the wavelengths are about 3.5 8.1 $R_{E}$. The lifetime of one tail flapping motion is about several hours. The tail flapping waves generally occur during quiet or weak activity periods. The locations of the observed tail flapping motions are about ten earth radii far away from the midnight meridian. These observations have shown that the tail flapping motions are large scale and long-lasting global processes, which may possibly be caused by the interaction between the solar wind and the magnetosphere when there are enhancements of the velocity or/and the dynamic pressure of the solar wind. The actual mechanism of the tail flapping waves and the source of their energy are still unclear. More observational and theoretical work is needed for a fuller understanding of the generation and evolution of tail flapping waves, and their roles on magnetospheric processes.

Acknowledgements. This work was supported by the National Natural Science Foundation of China Grant No. 40674094, and 40523006, Ministry of Science and Technology of China Grant 2006CB806305, the Hundred Talents Program of the CAS, International Space Science Institute at Bern, and the Specialized Research Fund for State Key Laboratories. The authors are grateful to Cluster FGM team, the Hungarian Data Centre and the ACE Science Center for providing us with four satellite magnetic measurement data, Cluster position data, and ACE level 2 data, respectively.

Topical Editor R. Nakamura thanks two anonymous referees for their help in evaluating this paper.

\section{References}

Angelopoulos, V., Kennel, C., Coroniti, F., Pellat, R., Kivelson, M., Walker, R., Russell, C., Baumjohann, W., Feldman, W., and Gosling, J.: Statistical Characteristics of Bursty Bulk Flow Events, J. Geophys. Res., 99, 21 257-21 280, 1994.

Asano, Y., Nakamura. R., Baumjohann, W., Runov, A., Vörös, Z., Volwerk, M., Zhang, T. L., Balogh, A., Klecker, B., and H. Rème: How typical are atypical current sheets?, Geophys. Res. Lett., 32, L03108, doi:10.1029/2004GL021834, 2005.

Balogh, A., Carr, C. M., Acuña, M. H., et al.: The Cluster magnetic field investigation: overview of in-flight performance and initial results, Ann. Geophys., 19, 1207-1217, 2001,

http://www.ann-geophys.net/19/1207/2001/.

Chanteur, G.: Spatial Interpolation for four spacecraft: Theory, in: Analysis Methods for Multi-Spacecraft Data, edited by: Paschmann, G. and Daly, P. W., p. 349, ESA Publications Division, Noordwijk, The Netherlands, 1998.

Dunlop, M. W., Balogh, A., Glassmeier, K.-H., and Robert, P.: Four-point Cluster application of magnetic field analysis tools: The Curlometer, J. Geophys. Res., 107, 1384 , doi:10.1029/2001JA005088, 2002.

Dunlop, M. W. and Woodward, T. I.: Multi-spacecraft discontinuity analysis: Orientation and motion, in: Analysis Methods for Multi-Spacecraft Data, edited by: Paschmann, G. and Daly, P. W., p. 271, ESA Publ. Div., Noordwijk, Netherlands, 1998.

Erkaev, N. V., Semenov, V. S., and Biernat, H. K.: Magnetic double gradient mechanism for flapping oscillations of a current sheet, Geophys. Res. Lett., 35, L02111, doi:10.1029/2007GL032277, 2008.

Escoubet, C. P., Fehringer, M., and Goldstein, M.: The Cluster mission, Ann. Geophys., 19, 1197-1200, 2001, http://www.ann-geophys.net/19/1197/2001/.

Fairfield, D. H.: A statistical determination of the shape and the position of the geomagnetic neutral sheet, J. Geophys. Res., 85, 775-780, 1980.

Fairfield, D. H., Hones, E. W., and Meng, C. I.: Multiple crossing of a very thin plasma sheet in the Earth's magnetotail, J. Geophys. Res., 86, 11 189-11200, 1981.

Golovchanskaya, I. V. and Maltsev, Y. P.: On the identification of plasma sheet flapping waves observed by Cluster, J. Geophys. Res., 32, L02102, doi:10.1029/2004GL021552, 2005.

Harris, E. G.: On a plasma sheet separating regions of oppositely directed magnetic field, Nuovo Cimento, 23, 115-121, 1962.

Harvey, C. C.: Spatial gradients and the volumetric tensor, in: Analysis Methods for Multi-Spacecraft Data, edited by: Paschmann, G. and Daly, P. W., p. 307, ESA Publications Division, Noordwijk, The Netherlands, 1998.

Kaymaz, Z., Siscoe, G., Luhmann, J. G., Fedder, J. A., and Lyon, J. G.: Interplanetary Magnetic Field Control of Magnetotail Field: IMP 8 Data and MHD Model Compared, J. Geophys. Res., 100, 17 163-17 172, 1995.

Liu, Z. X., Escoubet, C. P., Pu, Z., Laakso, H., Shi, J. Q., Shen, C., and Hapgood, M.: The Double Star Mission, Ann. Geophys., 23, 2707-2712, 2005, http://www.ann-geophys.net/23/2707/2005/.

Lui, A. T. Y., Meng, C.-I., and Akasofu, S.-I.: Wavy nature of the magnetotail neutral sheet, Geophys. Res. Lett., 5, 279-282, 1978.

Lui, A. T. Y.: Characteristics of the cross-tail current in the Earth's magnetotail, in: Magnetospheric Currents, AGU Geophysical 
Monograph, 28, 158-170, 1984.

Lui, A. T. Y.: Potential plasma instabilities for substorm expansion onsets, Space Sci. Rev., 113, 127-206, 2004.

Malova, H. V., Zelenyi, L. M., Popov, V. Y., Delcourt, D. C., Petrukovich, A. A., and Runov, A. V.: Asymmetric thin current sheets in the Earth's magnetotail, Geophys. Res. Lett., 34, L16108, doi:10.1029/2007GL030011, 2007.

McComas, D. J., Russell, C. T., Elphic, R. C., and Bame, S. J.: The Near-Earth Cross-Tail Current Sheet: Detailed ISEE1 and 2 Case Studies, Geophys. Res., 91, 4287-4301, 1986.

Nakamura, R., Baumjohann, W., Runov, A., and Asano, Y.: Thin Current Sheets in the Magnetotail Observed by Cluster, Space Sci. Rev., 122, 29-38, 2006.

Nakagawa, T. and Nishida, A.: Southward magnetic field in the neutral sheet produced by wavy motions in the dawn-dusk direction, Geophys. Res. Lett., 11, 1265-1268, 1989.

Ness, N. F.: The Earth's magnetic tail, J. Geophys. Res., 70, 29893005, 1965.

Petrukovich, A. A., Baumjohann, W., Nakamura, R., Runov, A., and Balogh, A.: Cluster vision of the magnetotail current sheet on a macroscale, J. Geophys. Res., 110, A06204, doi:10.1029/2004JA010825, 2005.

Petrukovich, A. A., Zhang, T. L., Baumjohann, W., Nakamura, R., Runov, A., Balogh, A., and Carr, C.: Oscillations of flux tube slippage in the quiet plasma sheet, Ann. Geophys., 24, 16951704, 2006, http://www.ann-geophys.net/24/1695/2006/.

Runov, A., Nakamura, R., Baumjohann, W., Zhang, T. L., Volwerk, M., Eichelberger, H. U., and Balogh, A.: Cluster observation of a bifurcated current sheet, Geophys. Res. Lett., 30(2), 1036, doi:10.1029/2002GL016136, 2003.

Runov, A., Sergeev, V. A., Baumjohann, W., Nakamura, R., Apatenkov, S., Asano, Y., Volwerk, M., Vörös, Z., Zhang, T. L., Petrukovich, A., Balogh, A., Sauvaud, J. A., Klecker, B., and Rème, H.: Electric current and magnetic field geometry in flapping magnetotail current sheets, Ann. Geophys., 23, 1391-1403, 2005a, http://www.ann-geophys.net/23/1391/2005/.

Runov, A., Sergeev, V. A., Nakamura, R., Baumjohann, W., Zhang, T. L., Asano, Y., Volwerk, M., Vörös, Z., Balogh, A., Sauvaud, J.-A., and Rème, H.: Reconstruction of the magnetotail current sheet structure using multi-point Cluster measurements, Planet. Space Sci., 53, 237-243, 2005b.

Runov, A., Sergeev, V. A., Nakamura, R., Baumjohann, W., Apatenkov, S., Asano, Y., Takada, T., Volwerk, M., Vörös, Z., Zhang, T. L., Sauvaud, J.-A., Rème, H., and Balogh, A.: Local structure of the magnetotail current sheet: 2001 Cluster observations, Ann. Geophys., 24, 247-262, 2006,

http://www.ann-geophys.net/24/247/2006/.

Russell, C. T., Mellott, M. M., Smith, E. J., and King, J. H.: Multiple spacecraft observations of interplanetary shocks: Four spacecraft determination of shock normals, J. Geophys. Res., 88, 4739-4748, 1983.

Schwartz, S. J.: Shock and discontinuity normals, Mach numbers, and related parameters, in: Analysis Methods for MultiSpacecraft Data, edited by: Paschmann, G. and Daly, P. W., p. 249, ESA Publ. Div., Noordwijk, Netherlands, 1998.

Sergeev, V. A., Mitchell, D. G., Russell, C. T., and Williams, D. J.: Structure of the tail plasma/current sheet at $11 \mathrm{Re}$ and its changes in the course of a substorm, J. Geophys. Res., 98, 17 345-17365, 1993.
Sergeev, V. A., Pulkkinen, T., and Pellinen, R.: Coupled-mode scenario for the magnetospheric dynamics, J. Geophys. Res., 101(A6), 13 047-13 065, 1996.

Sergeev, V. A., Runov A., Baumjohann,W., Nakamura, R., Zhang, T. L., Volwerk,M., Balogh, A., Rème, H., Sauvaud, J. A., Andrè, M., and Klecker, B.: Current sheet flapping motion and structure observed by Cluster, Geophys. Res. Lett., 30, 1327, doi:10.1029/2002GL016500, 2003.

Sergeev, V. A., Runov, A., Baumjohann,W., Nakamura, R., Zhang, T. L., Balogh, A., Louarn, P., Sauvaud, J.-A., and Rème, H.: Orientation and propagation of current sheet oscillations, Geophys. Res. Lett., 31, L05807, doi: 10.1029/2003GL019346, 2004.

Sergeev, V. A., Sormakov, D. A., Apatenkov, S. V., Baumjohann, W., Nakamura, R., Runov, A., Mukai, T., and Nagai, T.: Survey of large-amplitude flapping motions in the midtail current sheet, Ann. Geophys., 24, 2015-2024, 2006, http://www.ann-geophys.net/24/2015/2006/.

Shen, C., Li, X., Dunlop, M., Liu, Z. X., Balogh, A., Baker, D. N., Hapgood, M., and Wang, Xinyue: Analyses on the geometrical structure of magnetic field in the current sheet based on Cluster measurements, J. Geophys. Res., 108(A5), 1168, doi:10.1029/2002JA009612, 2003.

Shen, C., Li, X., Dunlop, M., Shi, Q. Q., Liu, Z. X., Lucek, E., and Chen, Z. Q.: Magnetic field rotation analysis and the applications, J. Geophys. Res., 112, A06211, doi:10.1029/2005JA011584, 2007a.

Shen, C., Dunlop, M., Li, X., Liu, Z. X., Balogh, A., Zhang, T. L., Carr, C. M., Shi, Q. Q., and Chen, Z. Q.: New approach for determining the normal of the bow shock based on Cluster four-point magnetic field measurements, J. Geophys. Res., 112, A03201, doi:10.1029/2006JA011699.2007b.

Shen, C. and Dunlop, M. W.: Geometric structure analysis of the magnetic field, in: Multi-Spacecraft Analysis Methods Revisited, edited by: Paschmann, G. and Daly, P. W., ISSI Science Report, SR-008, chapter 3, p. 27-32, Kluwer Academic Pub., 2008a.

Shen, C., Liu, Z., Li, X., Dunlop, M. W., Lucek, E. A., Rong, Z., Chen, Z., Escoubet, C. P., Malova, H. V., Lui, A. T. Y., Fazakerley, A. N., Walsh, A. P., and Mouikis, C.: Flattened Current Sheet and its Evolution in Substorms, J. Geophys. Res., 113, A07S21, doi:10.1029/2007JA012812, 2008b.

Shi, Q. Q., Shen, C., Pu, Z. Y., Dunlop, M. W., Zong, Q.-G., Zhang, H., Xiao, C. J., Liu, Z. X., and Balogh, A.: Dimensional analysis of observed structures using multipoint magnetic field measurements: Application to Cluster, Geophys. Res. Lett., 32, L12105, doi:10.1029/2005GL022454, 2005.

Shi, Q. Q., Shen, C., Pu, Z. Y., Dunlop, M. W., Zong, Q.G., Liu, Z. X., and Balogh, A.: Motion of observed structures calculated from multi-point magnetic field measurements: Application to Cluster, Geophys. Res. Lett., 33, L08109, doi:10.1029/2005GL025073, 2006.

Sibeck, D. G., Siscoe, G. L., Slavin, J. A., Smith, E. J., Tsurutani, B. T., and Lepping, R. P.: The distant magnetotail's response to a strong interplanetary magnetic field By - Twisting, flattening, and field line bending, J. Geophys. Res., 90, 4011-4019, 1985.

Sonnerup, B. U. Ö., Haaland, S. E., and Paschmann, G.: Discontinuity Orientation, Motion, and Thickness, in: Multi-Spacecraft Analysis Methods Revisited, edited by: Paschmann, G. and Daly, P. W., ISSI Science Report, SR-008, chap. 1, pp 1-15, Kluwer 
Academic Pub., 2008.

Speiser, T. W. and Ness, N. F.: The neutral sheet in the geomagnetic tail: its motion, equivalent currents, and field-line reconnection through it, J. Geophys. Res., 72, 131-141, 1967.

Speiser, T. W.: magnetospheric current sheets, Radio Sci., 8, 973977, 1973.

Thompson, S. M., Kivelson, M. G., Khurana, K. K., McPherron, R. L., Weygand, J. M., Balogh, A., Rème, H., and Kistler, L. M.: Dynamic Harris current sheet thickness from Cluster current density and plasma measurements, J. Geophys. Res., 110, A02212, doi:10.1029/2004JA010714, 2005.

Thompson, S. M., Kivelson, M. G., El-Alaoui, M., Balogh, A., Rème, H., and Kistler, L. M.: Bifurcated current sheets: Statistics from Cluster magnetometer measurements, J. Geophys. Res., 111, A03212, doi:10.1029/2005JA011009, 2006.

Toyichi, T. and Miyazaki, T.: Flapping motions of the tail plasma sheet induced by the interplanetary magnetic field variations, Planet. Space Sci., 24, 147-159, 1976.

Tsyganenko, N. A. and Fairfield, D. H.: Global shape of the magnetotail current sheet as derived from Geotail and Polar data, J. Geophys. Res., 109, A03218, doi:10.1029/2003JA010062, 2004.
Volwerk, M., Glassmeier, K.-H., Runov, A., Baumjohann, W., Nakamura, R., Zhang, T. L., Klecker, B., Balogh, A., and Rème, H.: Kink mode oscillation of the current sheet, Geophys. Res. Lett., 30, 1320, doi:10.1029/2002GL016467, 2003.

Zhang, T. L., Baumjohann, W., Nakamura, R., Balogh, A., and Glassmeier, K.-H.: A wavy twisted neutral sheet observed by Cluster, Geophys. Res. Lett., 29, 1899, doi:10.1029/2002/GL015544, 2002.

Zhang, T. L., Nakamura, R., Volwerk, M., Runov, A., Baumjohann, W., Eichelberger, H. U., Carr, C., Balogh, A., Sergeev, V., Shi, J. K., and Fornacon, K.-H.: Double Star/Cluster observation of neutral sheet oscillations on 5 August 2004, Ann. Geophys., 23, 2909-2914, 2005, http://www.ann-geophys.net/23/2909/2005/.

Zelenyi, L. M., Malova, H. V., Popov, V. Y., Delcourt, D., and Sharma, A. S.: Role of electrostatic effects in thin current sheets, in: Multiscale Processes in the Earth's Magnetosphere: From Interball to Cluster, edited by: Sauvaud, J.-A. and Nmecek, Z., Kluwer Academic Publisher, Netherland, 275-288, 2004. 